\title{
Conditions for coherent-synchrotron-radiation-induced microbunching suppression in multibend beam transport or recirculation arcs
}

\author{
C.-Y. Tsai, ${ }^{1,}{ }^{*}$ S. Di Mitri, ${ }^{2}$ D. Douglas, ${ }^{3}$ R. Li, ${ }^{1,3}$ and C. Tennant ${ }^{3}$ \\ ${ }^{1}$ Department of Physics, Virginia Tech, Blacksburg, Virginia 24061, USA \\ ${ }^{2}$ Elettra-Sincrotrone Trieste, 34149 Basovizza, Trieste, Italy \\ ${ }^{3}$ Jefferson Laboratory, Newport News, Virginia 23606, USA \\ (Received 15 November 2016; published 22 February 2017)
}

\begin{abstract}
The coherent synchrotron radiation (CSR) of a high-brightness electron beam traversing a series of dipoles, such as transport or recirculation arcs, may result in beam phase space degradation. On one hand, CSR can perturb electron transverse motion in dispersive regions along the beam line and possibly cause emittance growth. On the other hand, the CSR effect on the longitudinal beam dynamics could result in microbunching instability. For transport arcs, several schemes have been proposed to suppress the CSR-induced emittance growth. Correspondingly, a few scenarios have been introduced to suppress CSR-induced microbunching instability, which however mostly aim for linac-based machines. In this paper we provide sufficient conditions for suppression of CSR-induced microbunching instability along transport or recirculation arcs. Examples are presented with the relevant microbunching analyses carried out by our developed semianalytical Vlasov solver [C.-Y. Tsai, D. Douglas, R. Li, and C. Tennant, Linear microbunching analysis for recirculation machines, Phys. Rev. ST Accel. Beams 19, 114401 (2016)]. The example lattices include low-energy $(\sim 100 \mathrm{MeV})$ and high-energy $(\sim 1 \mathrm{GeV})$ recirculation arcs, and medium-energy compressor arcs. Our studies show that lattices satisfying the proposed conditions indeed have microbunching gain suppressed. Beam current dependences of maximal CSR microbunching gains are also demonstrated, which should help outline a beam line design for different scales of nominal currents. We expect this analysis can shed light on the lattice design approach that aims to control the CSR-induced microbunching.
\end{abstract}

DOI: 10.1103/PhysRevAccelBeams.20.024401

\section{INTRODUCTION}

Coherent synchrotron radiation (CSR) has been recognized as one of the most challenging issues for highbrightness beam transport line and recirculation machine designs, in which the beam phase-space quality is always aimed to preserve as well as possible before the beam fulfills its scientific mission or is transported to a subsequent beam line complex. CSR can be generated from electron coherent radiation emission inside a bend at a wavelength range comparable to the bunch length scale or to the order of the ripple density fluctuations atop. The radiation reaction can have effects on both transverse and longitudinal planes. In the transverse plane, because of the dispersive nature of a bending system, the energy change due to CSR can be correlated to the transverse coordinates ( $x$ or $x^{\prime}$ ) through the dispersion functions $R_{16}$ or $R_{26}$. Since the energy change varies for different slices of particles, such energy variation within a beam bunch can potentially

jcytsai@vt.edu

Published by the American Physical Society under the terms of the Creative Commons Attribution 4.0 International license. Further distribution of this work must maintain attribution to the author(s) and the published article's title, journal citation, and DOI. dilute the projected transverse emittance. In the longitudinal plane, initial small density modulations can be converted into energy modulations due to the tail-head collective interaction. Then the energy modulations can be transformed back to density counterparts downstream in dispersive regions via momentum compaction. The density-energy conversion, if forming a positive feedback in a multidipole system, can result in the enhancement of modulation amplitudes. This has been known as the CSR-induced microbunching instability [1-3].

Various mitigation techniques have been proposed to minimize or cancel the CSR-induced emittance growth, e.g. Refs. [4-12]. Correspondingly, a few different scenarios have been introduced to suppress CSR-induced microbunching instability, e.g. Refs. [13-17]. Among them, most of the CSR microbunching suppression mechanisms aim for linac-based machines, in which the number of dipoles is usually small (e.g. a two-dipole dogleg or four-dipole bunch compressor chicane). In this paper we provide a set of sufficient conditions for suppression of CSR-induced microbunching in arcs, which usually consist of several to tens of bending magnets. Our findings are therefore of interest for the design of magnetic lattices of recirculating accelerators, as well as of multidipole transport lines in single-pass facilities. It deserves here to further clarify the target of the beam line design. By recirculation here we 
mean a beam only passes through the arcs a few times. This is in contrast with storage-ring arcs, in which the assumption of an infinite number of beam passages is made. In addition, throughout the paper we only consider the steady-state CSR effect and for the moment exclude other effects such as transient CSR and space charge effects. The exit transient CSR (or CSR drift) in fact plays an equally (or even more) important role as it can further enhance the CSR microbunching gains (see, for example, Ref. [18]) in multibend transport arcs. The reason we assume only steady-state CSR interaction in this study is because of its simplicity; similar to discussion of suppression (or cancellation) of CSR-induced emittance growth [4-12], we always start from a simple consideration and later deal with transient effects.

In addition to CSR, the beam quality degradation can arise from the longitudinal space charge (LSC) effect. The LSC effect stems from upstream ripples on top of the bunch charge density and can accumulate an amount of energy modulation when the beam traverses a long section of a beam line. The LSC-induced microbunching can be as (or more) important as that induced by CSR, as had been emphasized in the existing literature (see, for example, Refs. [13,14]). In our subsequent analysis of conditions for microbunching suppression, the effect of LSC is neglected for the moment for the same reason as aforementioned. It however deserves here to comment the roles CSR and LSC play from the viewpoint of our analysis. By suppressing the CSR-induced microbunching instability we mean the goal to overcome the resultant (potentially large) microbunched density modulation due to initial bunch density modulation (in short, the density-to-density microbunching amplification). Different from CSR, which only occurs within bending magnets taking into account steady-state effect only, LSC is ubiquitous along a beam transport line. Therefore when the accumulation of energy modulation by LSC from the upstream section of beam line is significant, the downstream density modulation converted through the global momentum compaction $R_{56}(\mathrm{~s})$ (pure optics effect) can result in serious microbunching instability, in particular for nonisochronous arcs. This is referred to as the energy-to-density microbunching amplification. We note that the picture we just drew for energy-to-density modulation is only from a pure optics effect. More complete analysis should also include the effect of collective interaction and the information of upstream beam line lattice. The consideration of such energy-to-density configuration has however been beyond the scope of the paper. Apart from the energy to density, the resultant LSC-induced microbunching can also originate from density-to-density pathway, similar to CSR. For informative comparison, although we exclude LSC effect in the subsequent analysis (Sec. II), when we demonstrate numerical results in a set of our example lattices, we will show the optics impact on both mere CSR and integrated
(CSR-plus-LSC) effects that lead to density-to-density microbunching amplification.

In the remainder of this paper, we will begin, in Sec. II, from the integral equation that governs CSR microbunching instability, to outline the option of making small relative momentum compaction $R_{56}^{s_{1} \rightarrow s_{2}}$, and to discuss the conditions for CSR microbunching suppression in four situations: achromatic, dispersive, isochronous, and nonisochronous modules. Through the above analysis, it is found the kernel function plays a key role for microbunching gain development. In Sec. III, we examine these conditions by illustrating three sets of comparative examples, including low-energy $(\sim 100 \mathrm{MeV})$ and high-energy $(\sim 1 \mathrm{GeV})$ recirculation arcs, and medium-energy compressor arcs. Beam current dependences of maximal CSR microbunching gains are also demonstrated, which should help outline a beam line design for different current scales and give guidance of the level how small/large the relative momentum compaction should be retained. Finally, we conclude the results and comment on the difference of conditions between the transverse CSR emittance compensation and the longitudinal CSR microbunching suppression.

\section{CONDITIONS FOR CSR MICROBUNCHING SUPPRESSION}

Theoretical formulation of the CSR-induced microbunching instability based on the linearized Vlasov equation has been developed [2,3] in a single-pass beam transport system. The formulation assumes that the initial modulation wavelength is small compared with the whole bunch duration (i.e. coasting-beam approximation), consistent with most practical situations. In the linearized Vlasov treatment, the CSR effect is treated as a small perturbation to the beam phase space distribution. By the method of characteristics, the equation that governs the evolution of the complex bunching factor along a beam line can be written in the following integral equation:

$$
b(k ; s)=b_{0}(k ; s)+i \int_{0}^{s} d s^{\prime} \Lambda \mathcal{K}\left(s, s^{\prime}\right) b\left(k ; s^{\prime}\right),
$$

where $s^{\prime}$ denotes the location where CSR emission may occur and $s$ denotes the location where the beam bunch receives the energy kick. The bunching factors $b(k ; s)$ and $b_{0}(k ; s)$ are defined as the Fourier transformation of the longitudinal $z$ coordinate for perturbed and unperturbed (i.e. pure optics, in the absence of collective effect) phase space distributions, respectively. The coefficient $\Lambda$ characterizes the beam current and energy dependence,

$$
\Lambda=\frac{I_{b}}{\gamma I_{A}},
$$

where $\gamma$ is the relativistic factor, $I_{b}$ is the instantaneous beam current (depends on path length when bunch length 
varies), and $I_{A}$ is the Alfven current. The kernel function is particularly expressed as

$$
\mathcal{K}\left(s, s^{\prime}\right)=k(s) R_{56}^{s^{\prime} \rightarrow s} Z_{C S R}^{s s}\left(k ; s^{\prime}\right)\left\{L . D . ; s, s^{\prime}\right\},
$$

where, of our primary interest, only the one-dimensional steady-state free-space CSR impedance (per unit length) $[19,20]$ is considered (with the cgs unit)

$$
Z_{\mathrm{CSR}}^{s s}[k(s) ; s]=(1.63+0.94 i) \frac{k(s)^{1 / 3}}{|\rho(s)|^{2 / 3}},
$$

where $k=2 \pi / \lambda$ is the spatial wave number (with $\lambda$ the modulation wavelength), $\rho$ is the bending radius of a dipole, the transport matrix elements

$$
R_{56}^{s^{\prime} \rightarrow s}=\left[\mathbf{R}^{s^{\prime} \rightarrow s}\right]_{56}=\left[\mathbf{R}^{0 \rightarrow s}\left(\mathbf{R}^{0 \rightarrow s^{\prime}}\right)^{-1}\right]_{56}
$$

and the Landau damping term [3]

$$
\begin{aligned}
& \left\{L . D . ; s, s^{\prime}\right\} \\
& =\exp \left\{-\frac{k_{0}^{2} \varepsilon_{x 0} \beta_{x 0}}{2}\left[R_{51}\left(s, s^{\prime}\right)-\frac{\alpha_{x 0}}{\beta_{x 0}} R_{52}\left(s, s^{\prime}\right)\right]^{2}\right. \\
& \left.\quad-\frac{k_{0}^{2} \varepsilon_{x 0}}{2} R_{52}^{2}\left(s, s^{\prime}\right)-\sigma_{\delta 0}^{2} R_{56}^{2}\left(s, s^{\prime}\right)\right\}
\end{aligned}
$$

with $k_{0}=k(s=0)$. Here we note that the above expressions are applicable to combined-function dipoles. $\varepsilon_{x 0}$ is the horizontal geometric emittance, $\alpha_{x 0}$ and $\beta_{x 0}$ are the initial Twiss parameters, and $\sigma_{\delta 0}$ is the rms uncorrelated relative energy spread assuming Gaussian energy distribution. $R_{5 i}\left(s, s^{\prime}\right)=C(s) R_{5 i}(s)-C\left(s^{\prime}\right) R_{5 i}\left(s^{\prime}\right)$, where $i=1,2,6$, and $C(s)=\left[1-h R_{56}(s)\right]^{-1}$ is the bunch compression factor, and $h$ is the initial chirp of the beam (assuming $z>0$ for the bunch head). To quantify the microbunching, we define the amplification gain as modular ratio of bunching factors

$$
G_{f} \equiv\left|\frac{b\left(k ; s_{f}\right)}{b_{0}(k ; 0)}\right|,
$$

where $s_{f}$ is denoted the exit location of a beam line. When the gain due to CSR is much larger than unity in a beam line, the lattice is said to be susceptible to CSR microbunching instability. From Eq. (3), the cure of CSRinduced microbunching can be undertaken by either enhancing Landau damping or making the relative momentum compaction function as small as possible throughout the beam line. Here we take the latter. To proceed, let us introduce a parameter, which we will use to characterize the CSR effect in a beam line lattice [see also Eq. (3)]:

$$
\xi=\left|\max \left\{R_{56}^{s^{\prime} \rightarrow s}\right\} \frac{k_{0}^{1 / 3}}{\rho^{2 / 3}} \Delta L\right|,
$$

where $\Delta L$ is the effective distance of CSR interaction. It is evaluated to be the length between repetitive patterns of largest $\left|R_{56}^{s^{\prime} \rightarrow s}\right|$ in a beam line design (as we will demonstrate later), because they usually characterize the most contributed CSR microbunching gain development [21]. In case there is not a clear repetitive structure in $\left|R_{56}^{s^{\prime} \rightarrow s}\right|$, usually occurring in arc compressors, we estimate the effective distance as the width of the largest $\left|R_{56}^{s^{\prime} \rightarrow s}\right|$ block.

It is found that $\xi$, together with the concept of multistage CSR amplification [21], can be used to quantify the lattice impact of CSR microbunching. Note that $\xi$ is independent of beam properties. The dependence of beam parameters goes in $\Lambda$ and Landau damping terms through Eqs. (2) and (6). In the subsequent discussion of this section regarding conditions of suppression of CSR-induced microbunching gain, we have excluded the effect of Landau damping; we only aim to reach small $R_{56}^{s^{\prime} \rightarrow s}$ of beam line optics, thus small $\xi$, in order to eventually have small kernel function $\mathcal{K}$. However, when demonstrating example lattices in Sec. III, we include all relevant beam dynamics in the calculation of CSR microbunching gain for thorough consideration.

Our previous work [21] had indicated the important role of the relative momentum compaction $R_{56}^{s^{\prime} \rightarrow s}$ for CSR microbunching development. Local isochronicity associated to smallness of the dispersion function (hence small $\xi$ ) can result in effective CSR microbunching suppression. Here we try to relate this important quantity to lattice Twiss parameters using standard linear matrix analysis [22]. Our goals are to find explicit conditions for CSR microbunching suppression and to further evaluate the design performance of a beam line lattice for the particular issue. Below we consider a general beam line as shown in Fig. 1. We want to formulate in terms of typical Twiss parameters the relative momentum compaction function $R_{56}^{s_{1} \rightarrow s_{2}}$, where, inside the two dipoles, $s_{1}$ and $s_{2}$ are measured from entrances of their corresponding elem ents. Assume the bend plane of the beam line lattice lies in $(x, z)$. The four-by-four linear transport matrix from the CSR emission site $\left(s_{1}\right)$ to receiving site $\left(s_{2}\right)$ can be obtained by $\mathbf{R}^{\text {total }}=\mathbf{R}^{s_{2} \rightarrow L_{b}} \mathbf{R}^{s_{1} \rightarrow s_{2}} \mathbf{R}^{0 \rightarrow s_{1}}$, i.e.

$\mathbf{R}^{s_{1} \rightarrow s_{2}}=\mathbf{R}^{\text {dipole }}\left(\theta=-\frac{L_{b}-s_{2}}{\rho_{2}}\right) \mathbf{R}^{\text {total }} \mathbf{R}^{\text {dipole }}\left(\theta=\frac{-s_{1}}{\rho_{1}}\right)$,

where the transfer matrix for a sector dipole $\mathbf{R}^{\text {dipole }}$ can be expressed as [22]

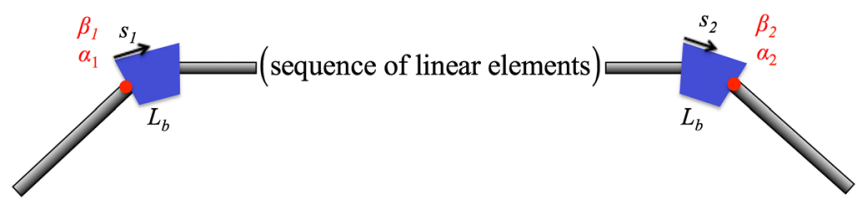

FIG. 1. Illustration of a two-dipole system. The in-between section can be a general transport section (see context for definition of notations). 


$$
\mathbf{R}^{\text {dipole }}(\theta)=\left[\begin{array}{cccc}
\cos \theta & \rho \sin \theta & 0 & \rho(1-\cos \theta) \\
-\frac{1}{\rho} \sin \theta & \cos \theta & 0 & \sin \theta \\
-\sin \theta & -\rho(1-\cos \theta) & 1 & -\rho(\theta-\sin \theta) \\
0 & 0 & 0 & 1
\end{array}\right]
$$

and the transfer matrix $\mathbf{R}^{\text {total }}$ in the two-dipole system can be in general parametrized by Twiss parameters [23]

$$
\mathbf{R}^{\text {total }}=\left[\begin{array}{cccc}
\sqrt{\frac{\beta_{2}}{\beta_{1}}}\left(\cos \psi_{21}+\alpha_{1} \sin \psi_{21}\right) & \sqrt{\beta_{2} \beta_{1}} \sin \psi_{21} & 0 & R_{16} \\
-\frac{1+\alpha_{2} \alpha_{1}}{\sqrt{\beta_{2} \beta_{1}}} \sin \psi_{21}+\frac{\alpha_{1}-\alpha_{2}}{\sqrt{\beta_{2} \beta_{1}}} \cos \psi_{21} & \sqrt{\frac{\beta_{1}}{\beta_{2}}}\left(\cos \psi_{21}-\alpha_{2} \sin \psi_{21}\right) & 0 & R_{26} \\
R_{51} & R_{52} & 1 & R_{56} \\
0 & 0 & 0 & 1
\end{array}\right],
$$

where $\alpha_{1}, \alpha_{2}, \beta_{1}$, and $\beta_{2}$ are assigned at locations illustrated in Fig. 1 . Note that the in-between section can be constituted by any sequence of linear elements, e.g. drifts, dipoles, quadrupoles, etc.

The symplecticity condition imposes the following constraints to the transport matrix [Eq. (11)]:

$$
R_{51}=R_{16} R_{21}-R_{26} R_{11} \quad R_{52}=R_{16} R_{22}-R_{26} R_{12} .
$$

Note that $R_{56}$ in Eq. (11) is considered a free parameter and independent of $R_{51}$ and $R_{52}$ (thus $R_{16}$ and $R_{26}$ ).

After some algebraic manipulation, the relative momentum compaction function $R_{56}^{S_{1} \rightarrow s_{2}}$ can be analytically obtained:

$$
\begin{aligned}
R_{56}^{s_{1} \rightarrow s_{2}}= & R_{56}+L_{b} \pm s_{1}-s_{2}+R_{26} \rho_{2}\left(\cos \frac{s_{2}-L_{b}}{\rho_{2}}-1\right) \mp \rho_{1} \sin \frac{s_{1}}{\rho_{1}}-R_{16} \sin \frac{s_{2}-L_{b}}{\rho_{2}}+\rho_{2} \sin \frac{s_{2}-L_{b}}{\rho_{2}} \\
& \mp \sin \frac{s_{1}}{\rho_{1}}\left\{-R_{26} \sqrt{\beta_{1} \beta_{2}} \sin \psi_{21}-\sqrt{\beta_{1} \beta_{2}} \sin \frac{s_{2}-L_{b}}{\rho_{2}} \sin \psi_{21}\right. \\
& \left.+\sqrt{\frac{\beta_{1}}{\beta_{2}}}\left(\cos \psi_{21}-\alpha_{2} \sin \psi_{21}\right)\left[R_{16}+\rho_{2}\left(\cos \frac{s_{2}-L_{b}}{\rho_{2}}-1\right)\right]\right\} \\
& +\rho_{1}\left(1-\cos \frac{s_{1}}{\rho_{1}}\right)\left\{\begin{array}{l}
-\sqrt{\frac{\beta_{2}}{\beta_{1}}}\left(\cos \psi_{21}+\alpha_{1} \sin \psi_{21}\right)\left(R_{26}+\sin \frac{s_{2}-L_{b}}{\rho_{2}}\right) \\
\left.-\frac{R_{16}+\rho_{2}\left(\cos \frac{s_{2}-L_{b}}{\rho_{2}}-1\right)}{\sqrt{\beta_{1} \beta_{2}}}\left[\left(\alpha_{2}-\alpha_{1}\right) \cos \psi_{21}+\left(1+\alpha_{1} \alpha_{2}\right) \sin \psi_{21}\right]\right\},
\end{array}\right.
\end{aligned}
$$

where the upper (lower) sign is for forward (reverse) bending.

As mentioned, to have effective suppression of CSR microbunching gain, one option we take is to pursue small $\xi$ (hence small $\mathcal{K}$ ) by manipulating $R_{56}^{s_{1} \rightarrow s_{2}}$. We remind that $R_{56}=0$ does not imply $R_{56}^{s_{1} \rightarrow s_{2}}=0$ and, if considering the transverse degrees of freedom, in general $R_{56}^{s_{1} \rightarrow s_{2}} \neq$ $R_{56}\left(s_{2}\right)-R_{56}\left(s_{1}\right)$ [see Eq. (5)]. To get the feeling of how $R_{56}^{s_{1} \rightarrow s_{2}}$ behaves in Eq. (13), we numerically illustrate the parametric dependencies of $R_{56}^{s_{1} \rightarrow s_{2}}$ for some specific cases. For simplicity, let us assume $\rho_{1}=\rho_{2}=10 \mathrm{~m}$, $L_{b}=1 \mathrm{~m}, \beta_{1}=\beta_{2}=\beta, \alpha_{1}=-\alpha_{2}=\alpha$, and $s_{1}$ and $s_{2}$ locate in the middle of dipoles. Given $R_{16}, R_{26}$, and $R_{56}$ for the two-dipole module (see Fig. 1), $R_{56}^{s_{1} \rightarrow s_{2}}$ depends only on lattice Twiss parameters $\alpha, \beta$ and phase difference $\psi_{21}$, i.e. $R_{56}^{s_{1} \rightarrow s_{2}}=f\left(\alpha, \beta, \psi_{21}\right)$. Figure 2 shows the typical $\left|R_{56}^{s_{1} \rightarrow s_{2}}\right|$ pattern for an achromatic $\left(R_{16}=R_{26}=0\right)$ and isochronous $\left(R_{56}=0\right)$ module. Note that in general $R_{56}^{s_{1} \rightarrow s_{2}}$ does not vanish even for an isochronous module. From the figure, it is obviously seen that small $\beta$ function is preferred. The choice of $\alpha$ function does not affect the resultant $\left|R_{56}^{s_{1} \rightarrow s_{2}}\right|$ too much in this specific parameter regime. The featured fact that $\psi_{21} \approx \pi$ (or its integer multiples) can lead to minimal $R_{56}^{s_{1} \rightarrow s_{2}}$ is also observed for all $\alpha$ 's and $\beta$ 's in Fig. 2.

The aforementioned observations from Fig. 2 turn out to be applicable to more general situations, for example, for dispersive and/or nonisochronous modules. To be concise in the main text, we make the supplementary discussion of 

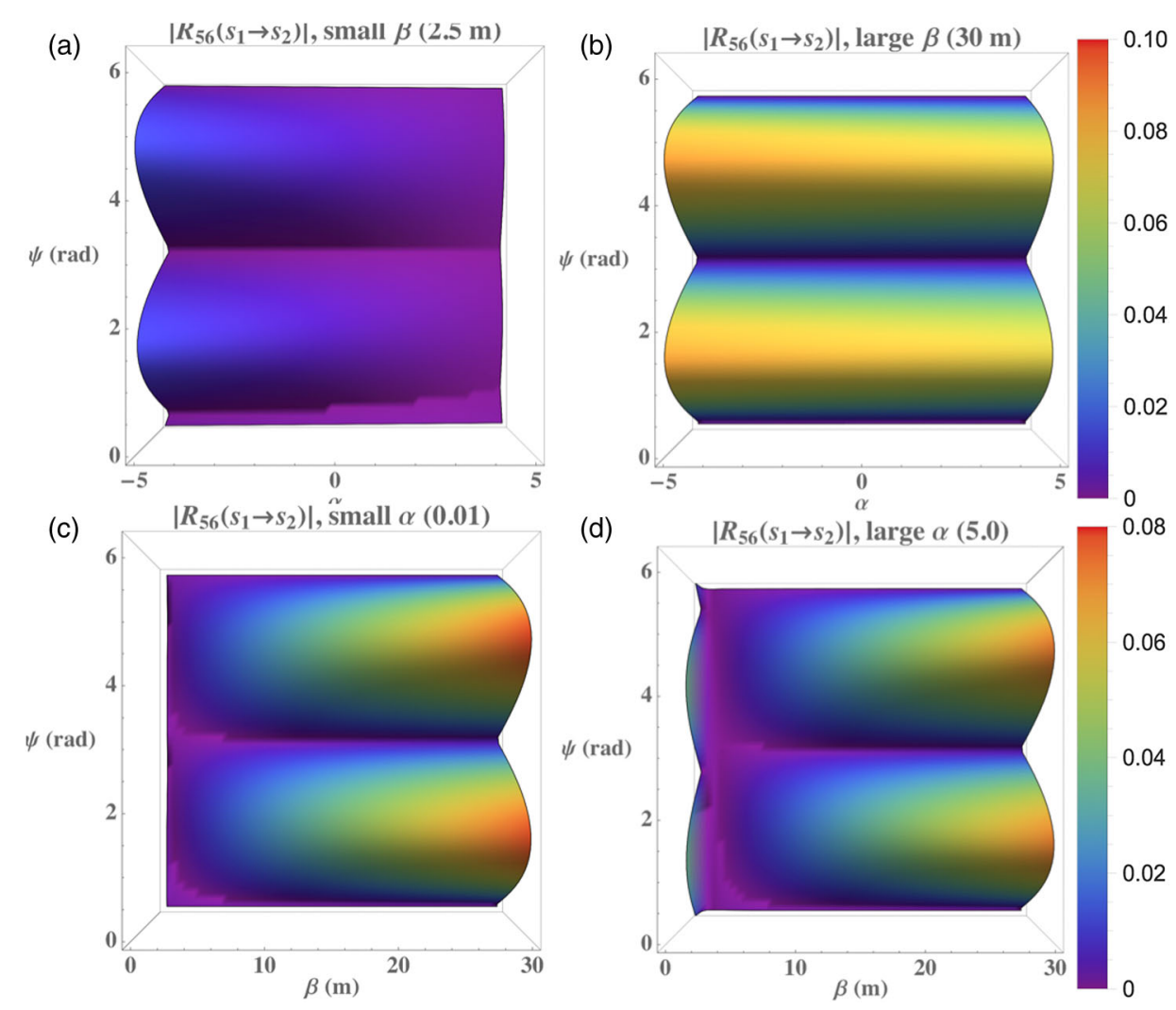

FIG. 2. $\left|R_{56}^{s_{1} \rightarrow s_{2}}\right|$ pattern for an achromatic and isochronous unit, $R_{16}=R_{26}=R_{56}=0$. (Top) $R_{56}^{s_{1} \rightarrow s_{2}}(\alpha, \psi)$ for (a) small and (b) large $\beta$ functions; (bottom) $R_{56}^{s_{1} \rightarrow s_{2}}(\beta, \psi)$ for (c) small and (d) large $\alpha$ functions. The color bar is measured in meters.

how $R_{56}^{s_{1} \rightarrow s_{2}}$ evolves when $R_{16} \neq 0, R_{26} \neq 0$, and/or $R_{56} \neq 0$ in the Appendix and only draw our conclusion here. After investigation of optical dependencies of the $R_{56}^{s_{1} \rightarrow s_{2}}$, we conclude that small $\beta$ functions (at entrances and exits of dipoles), moderate $\alpha$ functions (at entrances and exits of dipoles), and $\psi_{21} \approx \pi$ (or its integer multiples) between two relevant dipoles can in general lead to a small relative momentum compaction function in a general transport line. In a preliminary beam line design, Eq. (13) can serve as a way to check whether notional Twiss parameters (at entrances and exits of dipoles) are properly chosen to avoid being located at large $\left|R_{56}^{s_{1} \rightarrow s_{2}}\right|$. Whenever an optimal design is considered, one can in principle use Eq. (13) for more dedicated choices of Twiss functions and betatron phase advance. The small $\left|R_{56}^{S_{1} \rightarrow s_{2}}\right|$ (and hence small $\mathcal{K}$ ) will eventually bring about effective CSR microbunching suppression. Here we note that, although there is not a stability margin for $\left|R_{56}^{s_{1} \rightarrow s_{2}}\right|$ (or $\mathcal{K}$ ) in an absolute sense to have effective CSR gain suppression, since the collective interaction is intensity dependent (through $\Lambda$ ), our proposed optics conditions shall minimize the CSR-induced microbunching gain for any given beam current level. We also emphasize that these conditions are sufficient; other schemes of making small $\mathcal{K}$ are not excluded, e.g. Refs. [15-17] through enhancing Landau damping [see Eq. (3)].

\section{RECIRCULATION ARC EXAMPLES}

\section{A. High-energy recirculation arcs}

The first set of comparative examples involves two $1.3 \mathrm{GeV}$ high-energy recirculation arcs (hereafter named HERA v1 and v2 lattices). Figure 3 shows the optical Twiss functions and momentum compaction functions of the two arcs. Although both lattices have the same geometric layout, they exhibit distinct optical behaviors through properly tuning quadrupole focusing strengths. HERA v1 is a 180-deg arc with large momentum compaction. Moreover, it is a second-order achromat and globally isochronous with a large dispersion modulation across the entire arc [see Figs. 3(a) and 3(c)]. By contrast, HERA v2 is also a 180-deg arc with however small momentum compaction. This arc is also a second-order achromat but is designed to be a locally isochronous lattice within superperiods. Such local isochronicity ensures that the bunch length be kept the same at phase homologous CSR emission sites [see Figs. 3(b) and 3(d)]. Notably, it is shown in Figs. 3(c) and 3(d) that the momentum compaction function $R_{56}(s)$ for HERA v2 is considerably smaller in amplitude compared with that for HEAR v1 by at least 2 orders of magnitude due to local isochronicity. For the detailed description of the design for the two example lattices, we refer the interested reader to Ref. [9]. 

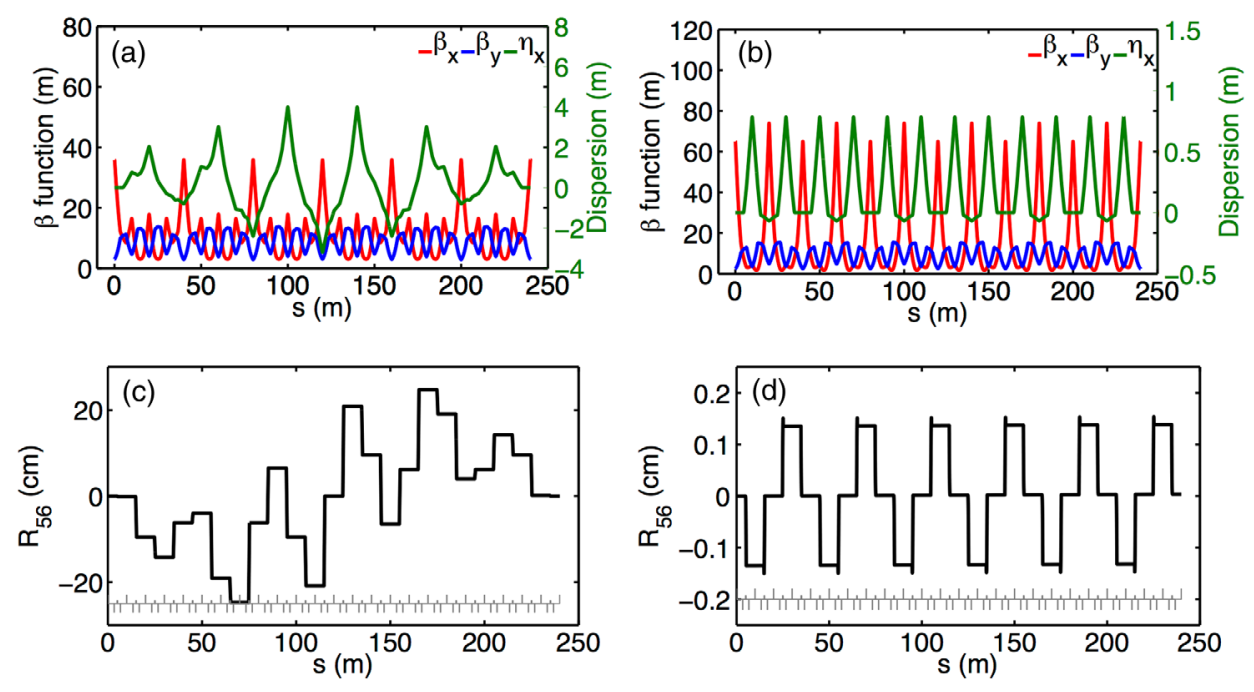

FIG. 3. Twiss functions and $R_{56}(\mathrm{~s})$ for HERA v1 [(a) and (c)] and v2 [(b) and (d)].

For both examples, the (peak) beam current is chosen to be $65 \mathrm{~A}$, the transverse normalized emittances are assumed $0.3 \mu \mathrm{m}$, and the uncorrelated energy spread is assumed $1.23 \times 10^{-5}$. These beam parameters are typical for nextgeneration light source facilities based on recirculation or energy recovery linacs (ERL) machines. Other relevant initial beam and lattice parameters are summarized in Table I. In this study, only steady-state CSR effect is considered and we will only briefly comment on the case with inclusion of all CSR effects (including entrance and exit/drfit transient effects) [18]. Figure 4 shows the nominal relative momentum compaction functions $R_{56}^{s^{\prime} \rightarrow s}$ for the two arc lattices. We remind of the difference between the pattern $R_{56}^{s^{\prime} \rightarrow s}$ in Fig. 4 and the variations of $R_{56}^{s_{1} \rightarrow s_{2}}$ in a range of Twiss parameters, shown in the Appendix. The former indicates how the CSR emission $\left(s^{\prime}\right)$ and receiving $(s)$ relate, and the latter demonstrates how $R_{56}^{s_{1} \rightarrow s_{2}}$ behaves as a function of varying Twiss parameters. Note that $R_{56}^{s^{\prime} \rightarrow s}$ plays the more essential role in determining the resultant microbunching gain than $R_{56}(s)$ does [see Eq. (3)]. This is because the energy modulation, induced by CSR within

TABLE I. Selected beam parameters used in HERA arcs.

\begin{tabular}{lccc}
\hline \hline Name & HERA v1 & HERA v2 & Unit \\
\hline Beam energy & 1.3 & 1.3 & $\mathrm{GeV}$ \\
Peak current & 65 & 65 & $\mathrm{~A}$ \\
Slice rms energy spread & $1.23 \times 10^{-5}$ & $1.23 \times 10^{-5}$ & \\
Normalized emittance & 0.3 & 0.3 & $\mu \mathrm{m}$ \\
Chirp & 0 & 0 & $\mathrm{~m}^{-1}$ \\
Compression factor & 1 & 1 & \\
$\rho$ & 3.6 & 3.6 & $\mathrm{~m}$ \\
$\Delta L$ & 40 & 20 & $\mathrm{~m}$ \\
$\max \left\{R_{56}\left(s^{\prime} \rightarrow s\right)\right\}$ & 0.52 & 0.13 & $\mathrm{~m}$ \\
$G_{f, \max }$ & 250 & 1 & \\
$\lambda_{\text {opt }}$ & 40 & 20 & $\mu \mathrm{m}$ \\
$\xi$ & 477.8 & 75.2 & $\mathrm{~m}$ \\
\hline \hline
\end{tabular}

dipoles, will be transformed back to density modulation downstream a beam line through the relative momentum compaction $R_{56}^{s^{\prime} \rightarrow s}$, instead of $R_{56}(s)=R_{56}^{0 \rightarrow s}$. The deep red blocks, considered as dangerous areas shown in Fig. 4 with larger $\left|R_{56}^{s^{\prime} \rightarrow s}\right|$, indeed result in enhancement of energy-todensity conversion and cause CSR microbunching instability. The quantity $\Delta L$, defined as the effective length of CSR interaction, can be evaluated from Fig. 4 as the edge-to-edge distance between adjacent large-amplitude (deep-red) blocks. For HERA v1, $\Delta L$ is roughly twice larger than that of HERA v2 (see Table I). To make $\Delta L$ small, it is preferred to design a beam line with more repetitive cell units, as the case of HERA v2.

As Eq. (5) shows, the momentum compaction function is indeed a continuous function, albeit Fig. 4 appears to feature a steplike diagram. This impression can be due to a mere visualization effect. As $R_{56}$ only varies within dipoles, it is not easy to observe the transition for the length of a beam line relatively larger than individual dipole lengths. For example, in Fig. 4 the dipole length for HERAv1 and v2 is $4.7 \mathrm{~cm}$ and the total length of the beam lines is $240 \mathrm{~m}$. While the
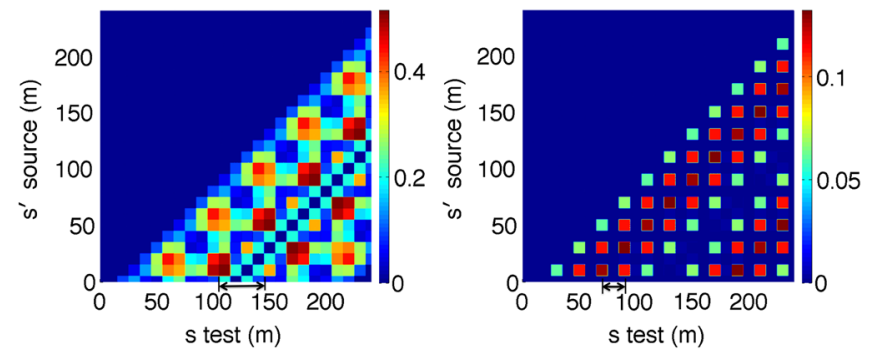

FIG. 4. Relative momentum compaction function $\left|R_{56}^{s / \rightarrow s}\right|$ for HERA v1 (left) and v2 (right). Note that $s^{\prime}$ is denoted as the source position and $s$ the observation or test position. The function is evaluated by Eq. (5). We quantify $\Delta L$ as $40 \mathrm{~m}$ and $20 \mathrm{~m}$ for HERA v1 and v2. The double-headed arrows indicate the effective length $\Delta L$. 

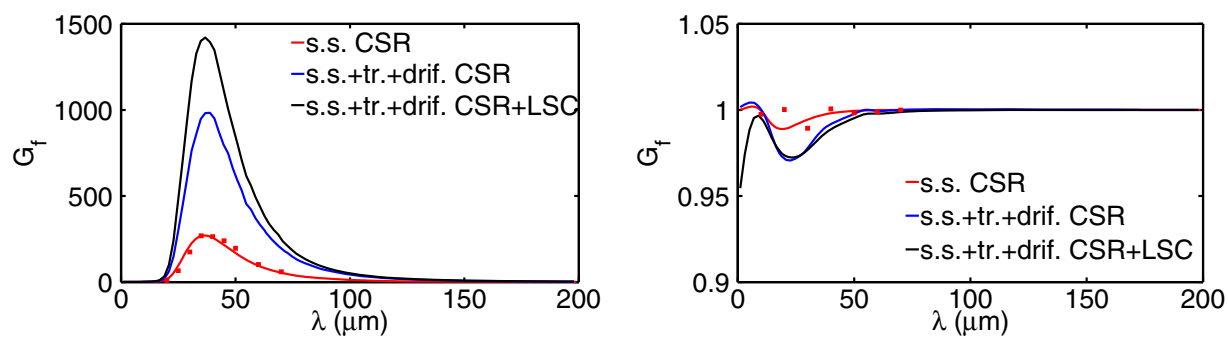

FIG. 5. Microbunching gain spectra for HERA v1 (left) and v2 (right) including steady-state (s.s.) only, steady-state plus entrance (tr.) and drift (drif.) transients, and the integrated (CSR-plus-LSC) effects. The dots are taken from particle tracking simulation by ELEGANT for steady-state CSR. For HERA v1, the dots are taken as the average over a set of data with initial modulation amplitude $0.05 \%$ and total of 70-million macroparticles are used. For HERA v2, the initial modulation amplitude is set $0.8 \%$ and the same number of macroparticles as HERA v1 is used. Detailed information of the impedance models of entrance transient and drift CSR and LSC effects can be found in Ref. [18].

momentum compaction (or the slippage) factor is often to characterize the one-turn $R_{56}$ effect in a storage-ring accelerator, in the single-pass system (e.g. in linear or recirculation accelerators) it was usually treated as a function of path length, as was dealt with in Fig. 1 of Ref. [2] for the study of CSR microbunching in the bunch compressor chicane of the linac coherent light source (LCLS).

Figure 5 shows the CSR-induced microbunching gain spectra (red curves and dots) for HERA v1 and v2 arcs, respectively. They were calculated with our developed semianalytical Vlasov solver [18]. A dramatic difference of CSR gain between the two examples is clearly indicated: HERA v1 is vulnerable to the CSR microbunching with peak gain $\sim 250$ whereas the microbunching gain in HERA v2 remains around unity (i.e. no microbunching amplification). To validate the Vlasov results, we benchmark the two example lattices by using
ELEGANT [24,25], with which extensive convergence studies were performed [26]. Both our Vlasov solution and ELEGANT tracking show excellent agreement in microbunching gain estimation. The reader is referred to Refs. [25,26] for more detailed and systematic convergence tests and postprocessing procedures to extract the microbunching gains from particle tracking data.

Our previous investigation [18] reveals that, with the inclusion of all relevant CSR effects (including steady-state and entrance and exit/drift CSR effects [27-29]), the microbunching gain of HERA v1 increases up to $400 \%$ compared with that of the steady-state case ( 250). Assume we turn on the longitudinal space charge (LSC) effect, we see from Fig. 5 that the microbunching gain of HERA v1 can further increase up to $600 \%$ in contrast to the steady-state CSR case. This indicates that, without optical compensation, the CSR exit/drift effect, as well as LSC, can

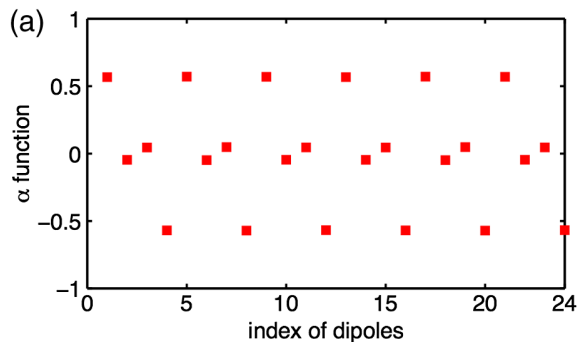

(c)

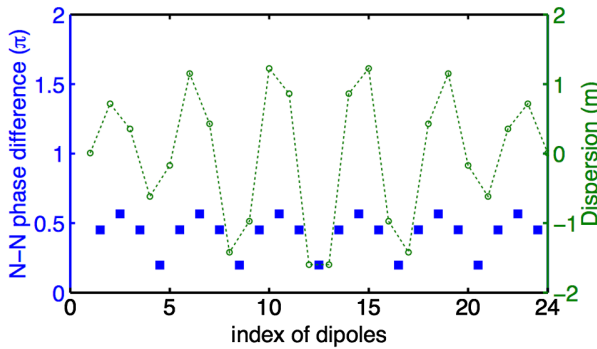

(b)

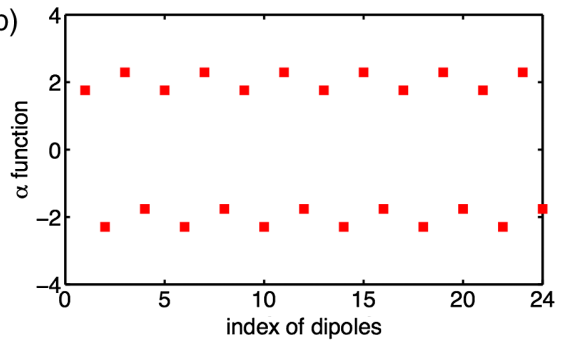

(d)

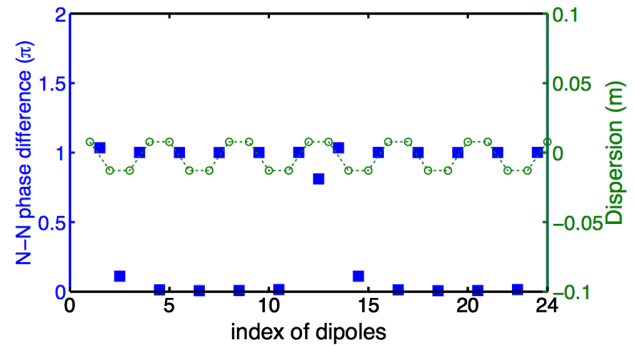

FIG. 6. Twiss $\alpha$ functions at dipoles and betatron phase differences $\psi_{21}$ (in unit of $\pi$ ) for HERA v1 [(a) and (c)] and v2 [(b) and (d)]. The dashed lines in (c) and (d) only help visualize the dispersion within dipoles and do not represent exact behavior of dispersion functions. 

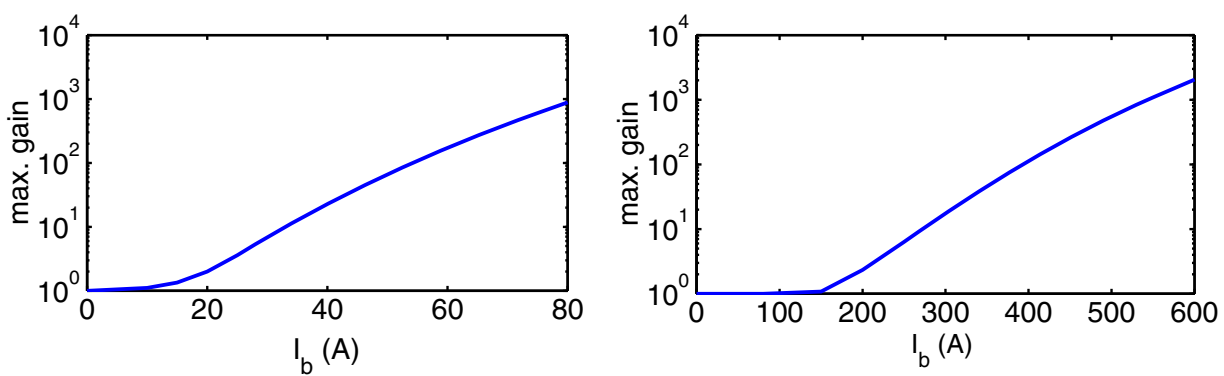

FIG. 7. Dependence of maximal CSR gains of HERA v1 (left) and v2 (right) on initial (peak) bunch current.

make a significant contribution on microbunching development. Yet with optical compensation, even with the same dipole configuration over the beam line, HERA v2 is neither subject to CSR nor LSC microbunching (the overall gains are still around unity even if all CSR and LSC effects are included). This highlights the impact of optical lattice design for recirculation arcs on microbunching gain development. In the analysis of this paper (Sec. II), we restrict our attention in the CSR-induced microbunching, for which the reason has been argued in Sec. I. We believe that the proposed optics conditions for suppression of CSR microbunching can work to the suppression of LSC microbunching as well, because we intend to minimize the kernel function $\mathcal{K}$ via $R_{56}^{s^{\prime}} \rightarrow s$ and do not limit the impedance to be CSR only. However, further investigation is certainly needed because more comprehensive analyses for LSC induced microbunching rely on the information of upstream beam line optics and the study of more aspects of phase space microbunched structures, e.g. energy-todensity amplification [30,31].

Next, we examine our proposed conditions in Sec. II for the two examples. Figures 6(a) and 6(b) compare Twiss $\alpha$ functions at dipole locations and betatron phase differences $\psi_{21}$ between near-neighbor dipoles. As mentioned, a moderate $\alpha$ function [Fig. 6(b)] is usually a good choice to produce small relative momentum compaction. The phase differences between near-neighbor dipoles for the two examples are illustrated in Figs. 6(c) and 6(d), respectively. HERA v2 with $\sim 0$ or $\sim \pi$ phase difference between adjacent dipoles indeed satisfies our proposed condition that smaller relative momentum compaction can be achieved when $\psi_{21} \approx \pi$ (or its integer multiples). In contrast, HERA v1 with $\psi_{21}$ scattering from 0 to $\pi / 2$ does not meet our proposed condition of phase difference. At dipoles, $\beta_{x} \leq 10 \mathrm{~m}$ for the two examples are comparable. The maximal magnitude of $R_{56}^{s^{\prime} \rightarrow s}$ for HERA v1 is 4 times larger than that of HERAv2. It is this difference in $R_{56}^{s^{\prime} \rightarrow s}$ that makes the distinct CSR microbunching development. To further evaluate the performance of the lattice designs, for each dipole pair as a twodipole module, we vary the nominal values of Twiss functions in a range and see where the design value of the momentum compaction $R_{56}^{s^{\prime} \rightarrow s}\left(\alpha_{x 0}, \beta_{x 0}, \psi_{21}\right)$ locates in the parameter space $\left(\alpha, \beta, \psi_{21}\right)$, where $\alpha \in\left(0.1 \alpha_{x 0}, 10 \alpha_{x 0}\right)$, $\beta \in\left(0.1 \beta_{x 0}, 10 \beta_{x 0}\right)$, and $\psi \in(0,2 \pi)$. Then we consider a pair to be dangerous once the nominal $R_{56}^{s_{1} \rightarrow s_{2}}\left(\alpha_{x 0}, \beta_{x 0}, \psi_{21}\right)$ is larger than $80 \%$ of the maximum value in the parameter space $\max \left\{R_{56}^{s_{1} \rightarrow s_{2}}(\alpha, \beta, \psi)\right\}$. Having examined all possible pairs of the two-dipole modules for HERA v1, we found 27 pairs (among a total of 276 combinations) are indicated as dangerous pairs and 12 of them are from near-neighbor dipoles. For HERA v2, all the near-neighbor pairs are evaluated to be safe.

The parameters $\Lambda$ and $\xi$ can be used to scale the maximal gain for comparative lattices. $\xi$ for HERA v1 is about 6.4 times larger than that of HERA v2. We see in Fig. 7 that indeed this multiplicative factor can reflect the scaling of bunch current for the maximal gains. This scaling can be used as a guideline of setting the order of magnitude of the maximal relative momentum compaction function $R_{56}^{s^{\prime} \rightarrow s}$ $($ via $\mathcal{K}$ ) for specified design goals. Assume we have already known the performance of HERA v2 and had no a priori detailed study of CSR microbunching about HERA v1. Now we want to estimate under what level of beam current the beam can be transported through HERA v1 with little CSR microbunching effect. Provided HERA v1 lattice is given, $\xi$ can be determined and compared with that of HREA v2. We then expect that a circulating beam with peak current 6 times smaller than that of the nominal one of HERA v1, i.e. $\sim 10$ A or 6 times smaller $\Lambda$, should not be

TABLE II. Selected beam parameters used in LERA arcs.

\begin{tabular}{lccc}
\hline \hline Name & LERA v1 & LERA v2 & Unit \\
\hline Beam energy & 150 & 100 & $\mathrm{MeV}$ \\
Peak current & 70 & 70 & $\mathrm{~A}$ \\
Slice rms energy spread & $2 \times 10^{-5}$ & $2 \times 10^{-5}$ & \\
Normalized emittance & 0.25 & 0.25 & $\mu \mathrm{m}$ \\
Chirp & 0 & 0 & $\mathrm{~m}^{-1}$ \\
Compression factor & 1 & 1 & \\
$\rho$ & 0.5 & 0.5 & $\mathrm{~m}$ \\
$\Delta L$ & 4 & 2 & $\mathrm{~m}$ \\
$\max \left\{R_{56}\left(s^{\prime} \rightarrow s\right)\right\}$ & 0.3 & 0.3 & $\mathrm{~m}$ \\
$G_{f, \max }$ & 34 & 1.2 & \\
$\lambda_{\text {opt }}$ & 68 & 104 & $\mu \mathrm{m}$ \\
$\xi$ & 86.1 & 37.4 & $\mathrm{~m}$ \\
\hline \hline
\end{tabular}



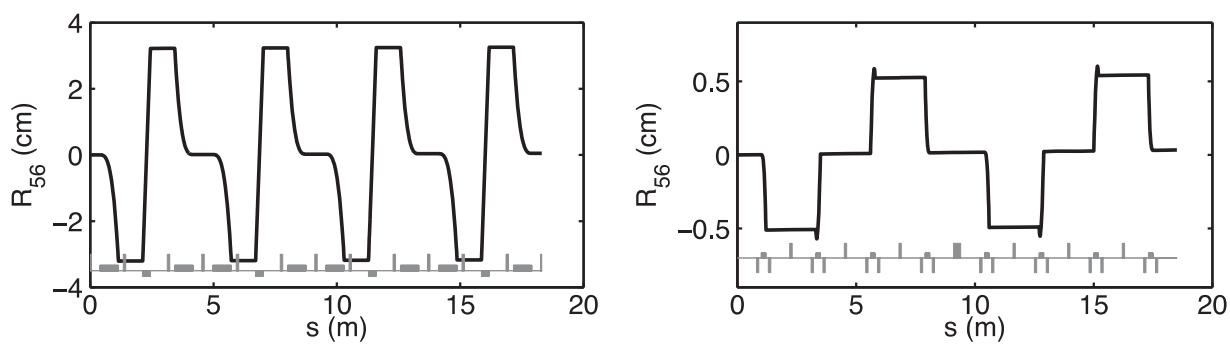

FIG. 8. $\quad R_{56}(\mathrm{~s})$ for LERA v1 (left) and v2 (right).

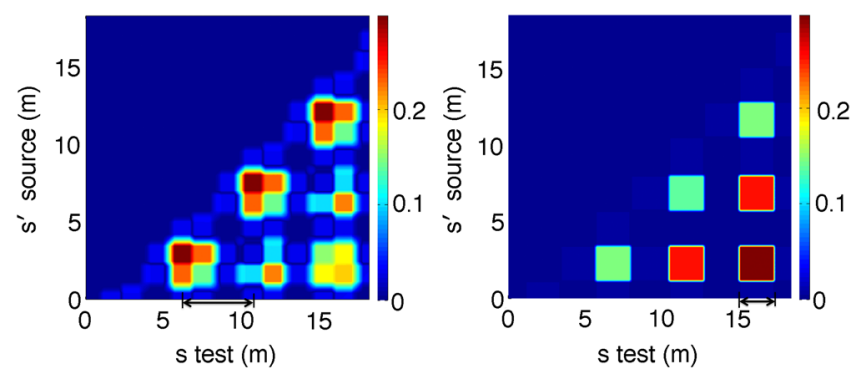

FIG. 9. Relative momentum compaction function $\left|R_{56}^{s / s}\right|$ for LERA v1 (left) and v2 (right). Note that $s^{\prime}$ is denoted as the source position and $s$ the observation or test position. We quantify $\Delta L$ as $4 \mathrm{~m}$ and $2 \mathrm{~m}$ for LERA $\mathrm{v} 1$ and $\mathrm{v} 2$. The double-headed arrows indicate the effective length $\Delta L$.

subject to CSR microbunching instability. This is immediately confirmed in the figure.

\section{B. Low-energy recirculation arcs}

The second set of examples consists of two low-energy recirculation arcs (LERA v1 and v2). LERA v1 is based on the design of an example arc outlined in Ref. [9] and proposed to serve as the return arc for a recirculating intrabeam scattering (RIBS) beam line [32]. LERA v2 is designed for a similar purpose to LERA v1 but more intended for mitigation of CSR microbunching instability. The design of LERA v2 also follows the guideline described in Ref. [9]. Both arcs are achromatic and (quasi)isochronous. Suppression of CSR-induced emittance growth has been taken care of for both examples (not shown here) [32]. Table II summarizes the relevant beam and lattice parameters and the featured parameters for the subsequent analysis.

Figure 8 illustrates the momentum compaction functions along the beam line $R_{56}(\mathrm{~s})$, in which the momentum compactions for the two lattices can be different by an order of magnitude. Figure 9 shows the quilt plots for LERA v1 and v2. As reasoned in the previous examples, the deep red blocks, with larger $R_{56}^{s^{\prime} \rightarrow s}$, indeed result in enhancement of energy-to-density conversion and can potentially cause CSR microbunching instability.

Figure 10 shows the CSR microbunching gain spectra for LERA v1 and v2, respectively, where we can see the gain for LERA v1 can be up to 35 at $\lambda=68 \mu \mathrm{m}$ while LERA v2 has nearly no gain $(\approx 1.2$ at $\lambda=104 \mu \mathrm{m})$. Our simulation results indicate that the gain can be up to 60 for LERA v1 and 1.4 for LERA v2 when including transient CSR effects (not shown here). This again confirms the importance of the optical compensation scheme employed in the beam line design. It is interesting to find that such LERA v1 design, which preserves the transverse beam emittance (when including CSR), does not necessarily ensure immunity from CSR microbunching instability. Filamentation of the longitudinal beam phase space distribution has been numerically observed and confirmed due to the CSR instability [32].

Now we examine our proposed conditions in Sec. II for the two lattices. Figures 11(a) and 11(b) compare Twiss $\alpha$ functions at dipole locations and betatron phase differences $\psi_{21}$ between near-neighbor dipoles. One can see from the top row of Fig. 11 that $\alpha$ 's for LERA v2 are in general larger
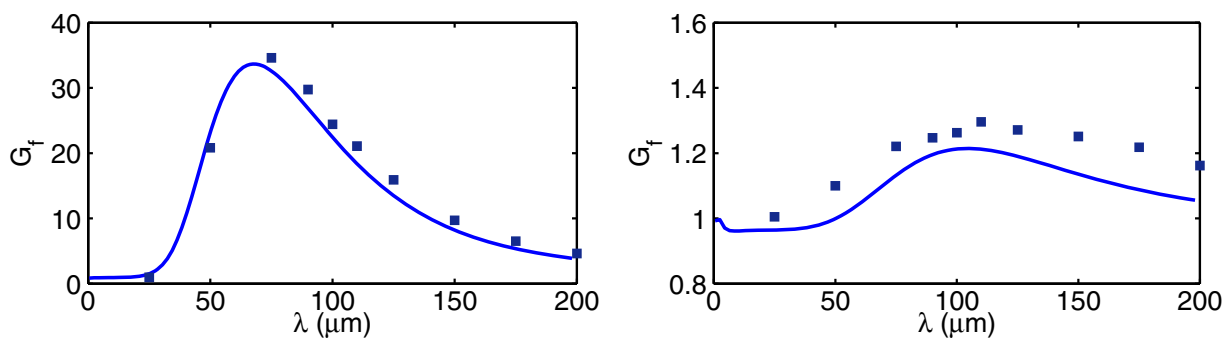

FIG. 10. CSR microbunching gain spectrum for LERA v1 (left) and v2 (right). Only steady-state CSR is included. The dots are taken from particle tracking simulation by ELEGANT, with a total of 40-million (with $0.1 \%$ initial modulation amplitude) and 10 -million (with $0.5 \%$ initial modulation amplitude) macroparticles used for LERA v1 and v2, respectively. 

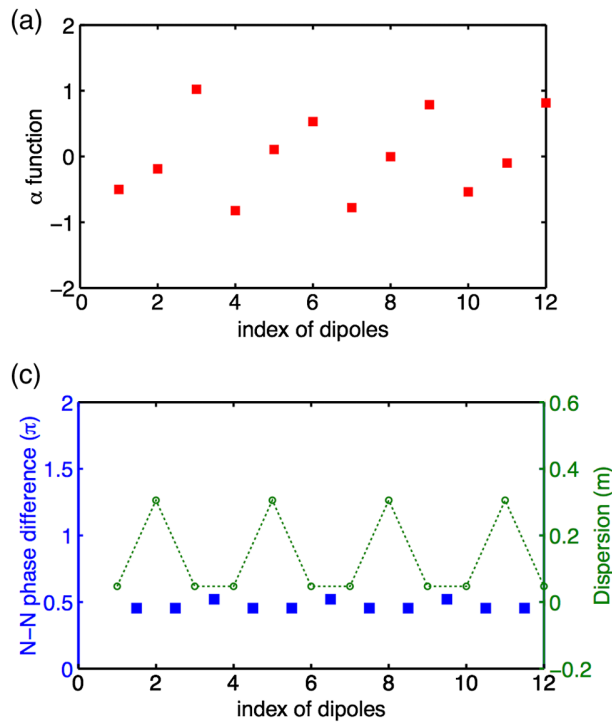
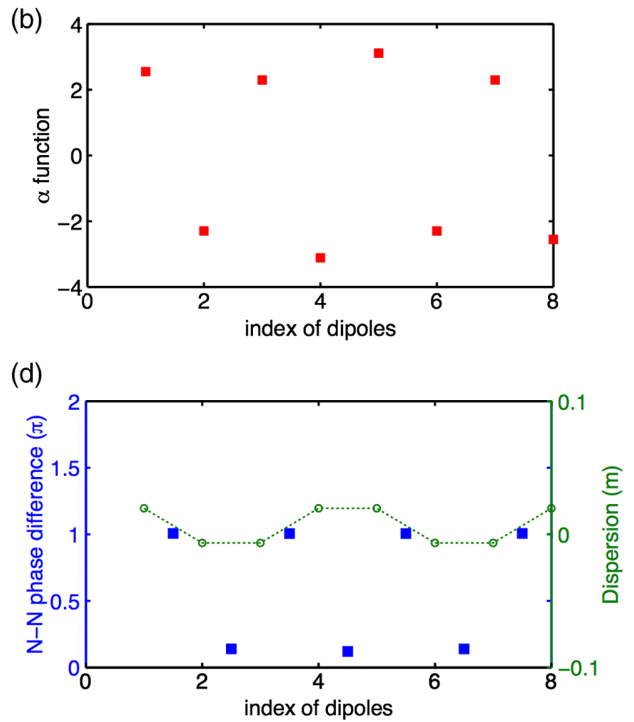

FIG. 11. Twiss $\alpha$ functions at dipoles and betatron phase differences $\psi_{21}$ (in unit of $\pi$ ) for LERA v1 [(a) and (c)] and v2 [(b) and (d)]. The dashed lines in (c) and (d) only help visualize the dispersion within dipoles and do not represent exact behavior of dispersion functions.
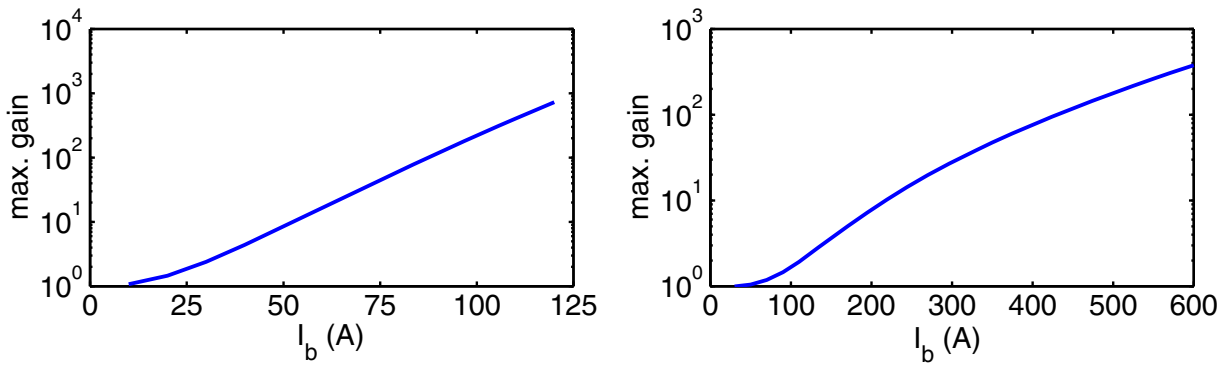

FIG. 12. Dependence of maximal CSR gains on (peak) bunch current for LERA v1 (left) and v2 (right).

than for LERA v1. The phase differences between nearneighbor dipoles for the two examples are illustrated in Figs. 11(c) and 11(d), respectively. LERA v1 with $\psi_{21} \approx$ $0.5 \pi$ does not meet our proposed condition of phase difference with $\pi$. In contrast, LERA v2 with $\sim 0$ or $\sim \pi$ phase difference between adjacent dipoles is favored based on our previous analysis. Although the maximum values of $R_{56}^{s^{\prime} \rightarrow s}$ are comparable for LERA v1 and v2, the deep-red blocks in LERA v1 can accumulate up to 3 times (of energy-density conversion) while it is only effective at one time for LERA v2 [23]. For the two examples, their averaged $\beta$ functions at dipoles $\left(\beta_{x} \leq 2\right)$ are comparable (not shown here). From Fig. 11, we conclude that the LERA v2 lattice meets our proposed conditions and thus has CSR gain suppressed. Moreover, for LERA v1, we found six pairs (among a total of 66 combinations) are indicated as dangerous pairs. For LERA v2, all the pairs are considered to be safe.

Similar to HERA examples, the scaling of $\Lambda$ is still followed for LERA arcs. For LERA v1, $\xi$ is about 3.5 times
TABLE III. Selected beam parameters used in compressor arcs.

\begin{tabular}{lccc}
\hline \hline & $\begin{array}{c}\text { FODO } \\
\text { compressor } \\
\text { arc }\end{array}$ & $\begin{array}{c}\text { Modulated } \\
\text { compressor } \\
\text { arc }\end{array}$ & Unit \\
\hline Name & 0.71 & 0.75 & $\mathrm{GeV}$ \\
Beam energy & $7.9,405$ & $7.9,405$ & $\mathrm{~A}$ \\
Peak current & & & \\
$\quad$ (before, after) & $1.13 \times$ & $1.13 \times$ & \\
Slice rms energy & $10^{-5}$ & $10^{-5}$ & \\
$\quad$ spread & 0.75 & 0.75 & $\mu \mathrm{m}$ \\
Normalized & & & \\
$\quad$ emittance & -1.577 & -0.6344 & $\mathrm{~m}^{-1}$ \\
Chirp & 51.26 & 51.26 & \\
Compression & & & \\
$\quad$ factor & 2 & 2 & $\mathrm{~m}$ \\
$\rho \quad 2.71$ & 2.36 & $\mathrm{~m}$ \\
$\Delta L$ & 0.62 & 1.6 & $\mathrm{~m}$ \\
$\max _{\text {max }}\left\{R_{56}\left(s^{\prime} \rightarrow s\right)\right\}$ & 2.8 & 2.2 & \\
$G_{f, \max }$ & 1800 & 4400 & $\mu \mathrm{m}$ \\
$\lambda_{\text {opt }}$ & 16.1 & 26.8 & $\mathrm{~m}$ \\
\hline
\end{tabular}



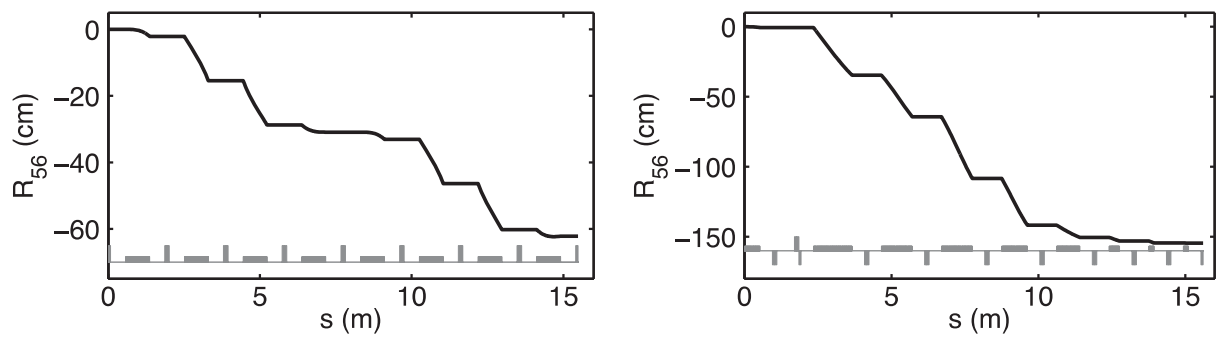

FIG. 13. $R_{56}$ (s) for FODO compressor arc (left) and modulated compressor arc (right).

larger than that of LERA v2 when beam energy is scaled to the same for both examples. We see in Fig. 12 that indeed the maximal gains follow the scaling of $\Lambda$. In view of the beam current scaling (together with Fig. 7), we can think of HERA v2 (LERA v2) as 6.4 (3.5) times effective suppression of CSR microbunching than HERA v1 (LERA v1).

\section{Medium-energy compressor arcs}

As the third set of examples, we consider two arcs that compress a high-brightness electron beam with nearly no beam quality degradation: the focusing-dipole-defocusingdipole (FODO) compressor arc and the modulated compressor arc [33]. The FODO compressor arc, considered as a conventional beam line design, consists of eight $\pi / 2$ FODO cells. All the bending radii are $2 \mathrm{~m}$ and form a 180-deg arc. After careful examination and optimization of phase-space distortion subject to CSR, the CSR-induced emittance growth, though difficult to exactly cancel, can be minimized [33]. The modulated compressor arc, a novel beam line design, is made up of total nine combined-function dipoles. Those dipoles are no longer constant-angle dipoles but wittingly designed with gradually reduced bending angles in order to avoid the increasingly detrimental CSR effects among the downstream bending magnets. The design strategy is based on the following physical intuitions. First, the energy change due to CSR effect is proportional to the CSR force (in steady state) $F^{\mathrm{CSR}}$ multiplied by the length of the dipole $\Delta s$. Decreasing $\Delta s$ may reduce the CSR effect, though the gradually compressed electron bunch will result in the increase of $\Lambda$ and enhance CSR microbunching. Second, when the dipole length becomes shorter than the overtaking distance [19,27], the transient effect appears and tends to make CSR not be formed timely. We find that such design strategy effectively alleviates the CSR-induced microbunching instability. The initial beam and lattice parameters for the two compressor arcs are tabulated in Table III. Figure 13 shows the momentum compaction functions $R_{56}(\mathrm{~s})$ of the two arc compressors. To compare, the initial beam parameters of the two arcs, except for bunch chirp, are assumed the same, and the output beams are compressed by the same ratio.

In Table III, the parameter $\xi$ is found to be comparable for the two arc designs; we expect the effect of steady-state CSR microbunching will be comparable as well $(\Lambda$ is the same). To examine the energy-density conversion, Fig. 14 shows the $R_{56}^{s^{\prime} \rightarrow s}$ quilt plots for the two arcs. The two quilt patterns are found to be similar, although the FODO compressor arc features smaller relative momentum compaction. The average $\beta$ functions are about 1 and $2.5 \mathrm{~m}$ respectively for FODO and modulated compressor arcs, both of which are within the preferred range. Figure 15 shows Twiss $\alpha$ functions and betatron phase differences $\psi_{21}$ of near-neighbor dipoles. Except the first pair, although $\psi_{21} \approx 0.5 \pi$ for the modulated arc compressor, considered not favored, it does not hit the dangerous region much. Our analysis shows that there are two near-neighbor dipole pairs in which $R_{56}^{s^{\prime} \rightarrow s}$ locate within dangerous areas for both compressor arcs.

The steady-state CSR microbunching gain spectra for the two arc compressors are presented in Fig. 16. The overall gains smaller than 3 indicate that the steady-state CSR does not have a significant effect on the two arcs. Though not shown here, our Vlasov calculation suggests the maximum CSR gain $\sim 15$ for FODO compressor arc and $\sim 3$ for modulated arc when including free-space transient CSR effects [34]. The CSR gain is commonly considered to be small below 10, so the modulated compressor arc appears to be a better design in this aspect. Note that the arc compressors presented here can be different from those specified as four-dipole compressor chicanes in that the arc compressors can have much larger $R_{56}$ so that the typical microbunched structure $(\sim 1 \mathrm{~mm})$ is usually much larger than that of typical bunch compressor chicanes $(\sim 50 \mu \mathrm{m})$.
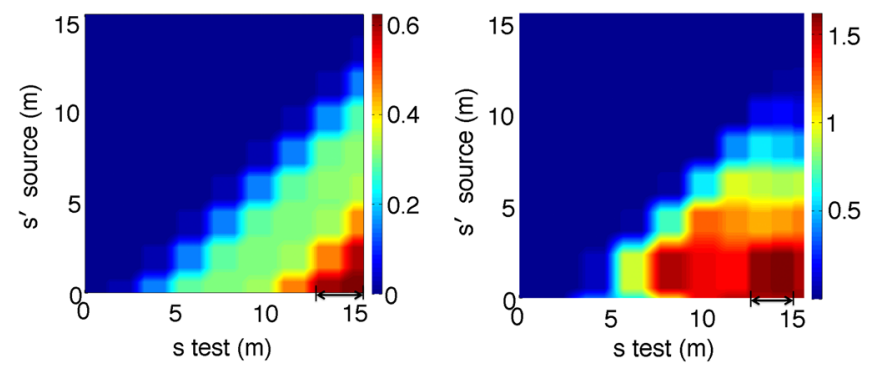

FIG. 14. Relative momentum compaction function $\left|R_{56}^{s / \rightarrow s}\right|$ for FODO compressor arc (left) and modulated compressor arc (right). Note that $s^{\prime}$ is denoted as the source position and $s$ the observation or test position. $\Delta L$ is approximately quantified to be 2.7 and $2.4 \mathrm{~m}$ for FODO and modulated arcs, though the periodicity of the quilt patterns is not obvious here. 

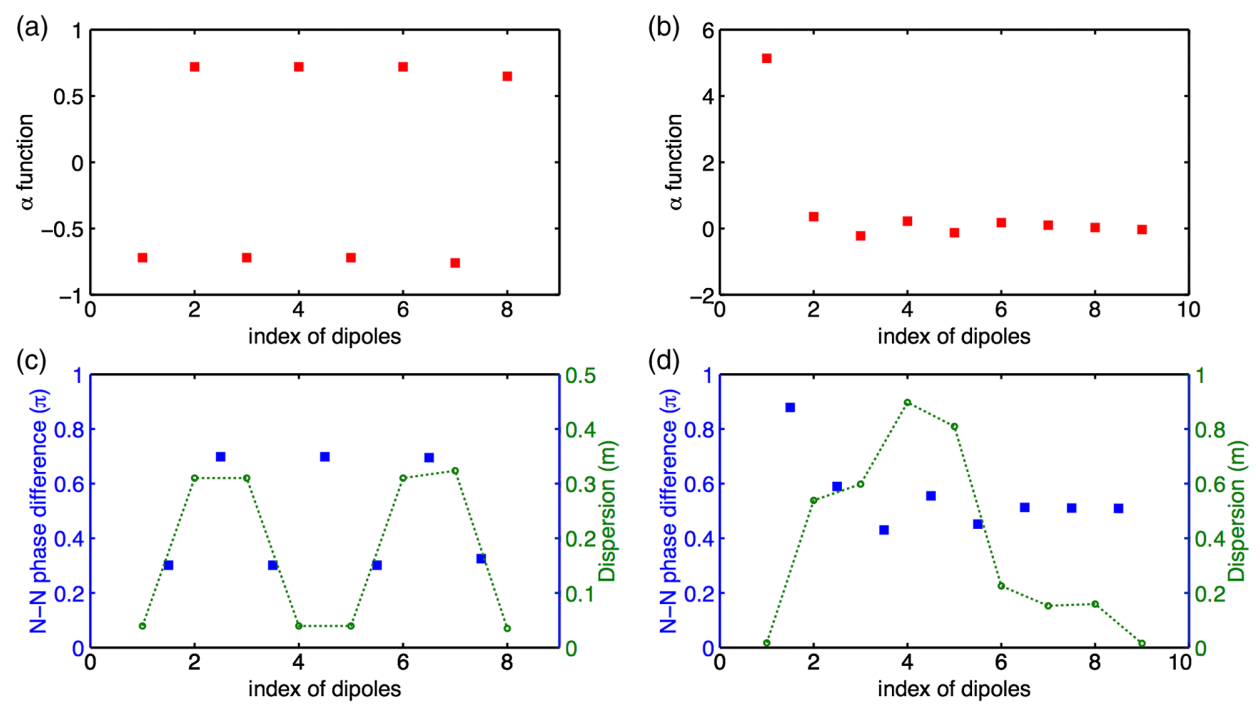

FIG. 15. Twiss $\alpha$ functions at dipole locations and betatron phase differences $\psi_{21}$ (in unit of $\pi$ ) for FODO compressor arc [(a) and (c)] and modulated compressor arc [(b) and (d)]. The dashed lines in (c) and (d) only help visualize the dispersion within dipoles and do not represent exact behavior of dispersion functions.
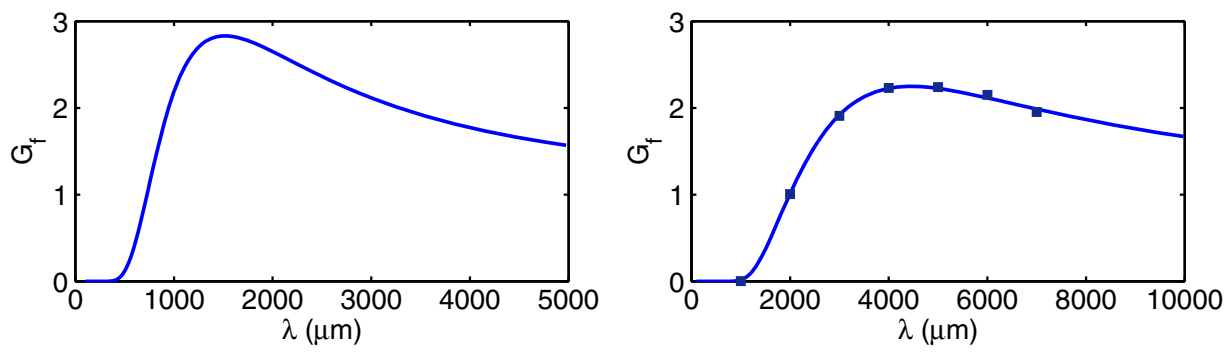

FIG. 16. CSR microbunching gain spectrum for FODO compressor arc (left) and modulated compressor arc (right). Note that only steady-state CSR is included. The dots in the right figure are taken from ELEGANT by tracking a total of 60-million macroparticles with the initial modulation amplitude set $1 \%$.

\section{SUMMARY}

In this paper we have applied our recently developed semianalytical Vlasov solver to study CSR-induced microbunching instability in various recirculation arc designs, including low-energy and high-energy recirculation arcs and medium-energy compressor arcs. We proposed and verified the validity of sufficient conditions for effective suppression of CSR-induced microbunching gains in recirculation arcs. The conditions of preferring small $\beta$ functions at dipoles and of keeping $\psi_{21} \approx m \pi$, with $m$ to be an integer, in consecutive two-dipole modules, aim for minimizing $\xi$ (hence the kernel function $\mathcal{K}$ ) through the relative momentum compaction function $R_{56}^{s_{1} \rightarrow s_{2}}$ in a beam line design. Those conditions apply to both periodic and nonperiodic magnetic lattices, from low- to high-energy regimes, and for constant as well as varying bunch length along a beam line. In the most general case of nonachromatic and nonisochronous beam line, the constraint of betatron phase difference can be weakened (other values slightly different from $m \pi$ may be preferred, e.g. see
Figs. 19 to 21), depending on the specific lattice design. In that case, Eq. (13) can be used to examine or optimize a beam line design when CSR microbunching becomes a concern.

Since the CSR-induced instability is intensity dependent, our proposed conditions can minimize the CSR-induced microbunching for any given electron bunch peak current. The three sets of example lattices can prove useful for beam line designs at difference energies or bunch currents through the scaling parameter $\Lambda$. We have thus investigated the beam current scaling of maximal CSR gains, and it can serve as a guideline of setting the maximal magnitude of the relative momentum compaction to be designed for similar purposes at a different level of bunch currents or beam energies.

We found that, in general, our proposed conditions can be different from the conditions for cancellation or minimization of CSR-induced emittance [11,12]. For example, compensation of CSR-induced emittance growth usually features some symmetry and can be achieved by separating dipoles (where CSR kicks occur) by $\pi$ cell by cell, but not 
necessarily needs to be for every dipole pairs, as intended in our situation. In this sense, our proposed conditions appear stronger, and, in principle, would result in emittance preservation under the CSR effect. In addition, CSRinduced microbunching features multistage amplification [21], as shown in Figs. 4 and 9. To avoid accumulation of effects from those deep-red blocks (i.e. strong energydensity conversion), a lattice with more repetitive cell units (i.e. local isochronicity) can lead to smaller momentum compaction and is therefore preferred. We also note that this study is to some extent empirical and qualitative. In fact, since the CSR-induced microbunching instability is intensity dependent and behaves as an amplifier [35] in a single-pass system unlike the case in storage-ring accelerators, there is not a clear threshold for a lattice design to be immune from the effect.

Last but not least, in the beam lines of interest here, we know that the LSC induced microbunching can be as important as, or even more important than, that induced by CSR, and eventually dominates the overall microbunching, for example, HERA v1 shown in Fig. 5. We believe the proposed optics conditions for suppression of CSR microbunching can work to the suppression of LSC microbunching as well because we intend to minimize the kernel function $\mathcal{K}$ via $R_{56}^{s^{\prime} \rightarrow s}$ and do not fully exclude the impedance term other than CSR. Although our previous studies $[18,36]$ support this statement, further investigation is certainly necessary because we have thus far only considered density-to-density modulation and, for LSC in particular, the upstream accumulated energy modulation can potentially lead to serious microbunching due to pure optics transport via global momentum compaction $R_{56}^{0 \rightarrow s}$ (instead of $R_{56}^{s^{\prime} \rightarrow s}$ ) and to collective interactions (through $R_{56}^{s^{\prime} \rightarrow s}$ ) [31]. The issue of LSC induced microbunching can become a serious concern when designing a nonisochronous arc because of unavoidable $R_{56}$ of the whole beam line. It may be envisioned that, if the proposed optics conditions can be taken care of for each pair of dipoles, the resultant microbunching development, at least the contribution by collective interaction, can be minimized. In addition, the inclusion of more aspects of microbunching dynamics will be a crucial step to proceed with a more complete analysis. At any rate, the proposed conditions here have to be included in the first-iterative beam line designs, similar to the situation of the conditions for suppression or cancellation of CSR-induced emittance growth. Recently, we have noticed that a potentially new mechanism, other than what Eq. (3) depicts, can drive microbunching instability as well, which is beyond the scope of our current study. Such a mechanism, lately identified in Ref. [37], is due to projection of space charge force on transverse-longitudinal dimension $(x, z)$ and is numerically observed to result in longitudinal microbunching amplification in LCLS-II dogleg design. How this transverse space-charge (TSC) induced microbunching can impact recirculating beam lines and whether our proposed conditions for suppression of CSR still work for this TSC microbunching deserves further investigation and would be in our plan.

Finally we note that, being the proposed conditions sufficient, they do not exclude other possible mitigation schemes.

\section{ACKNOWLEDGMENTS}

The authors would like to thank Steve Benson for reading and revising an early version of the manuscript. One of the authors (C. Y. T.) would like to thank IPAC 2016 student grant committee and his advisor at Virginia Tech, Professor Mark Pitt, for the travel support. This work is supported by Jefferson Science Associates, LLC under U.S. DOE Contract No. DE-AC05-06OR23177.

\section{APPENDIX: DISCUSSION OF RELATIVE MOMENTUM COMPACTION FUNCTION ON TWISS PARAMETERS}

In the main text we have discussed the simplest case with the achromatic and the isochronous module (Fig. 2). When the two-dipole module is dispersive (i.e. $R_{16} \neq 0$, while assuming $R_{26}=R_{56}=0$ ), the typical $\left|R_{56}^{s_{1} \rightarrow s_{2}}\right|$ patterns become distorted, as shown in Fig. 17. Our investigation finds that the effect of nonzero $R_{16}$ can create low-lying areas in some regions, as shown in Figs. 17(a) and 17(b). Small $\left|R_{56}^{S_{1} \rightarrow s_{2}}\right|$ however can still occur around $\psi_{21} \approx \pi$ except at $\alpha \approx 0$. The action of nonzero $R_{56}$ is to have either the upper or the lower $\left(\psi_{21}>\pi\right.$ or $\left.\psi_{21}<\pi\right)$ portion shrink asymmetrically, depending on the sign of $R_{56}$, as illustrated in Fig. 18 with positive $R_{56}$. Figure 19 demonstrates the action of $R_{26}$, which tends to restore the shape to that as Fig. 2 shows (see also Fig. 18); if $R_{26}$ continues to increase, Fig. 19 would reduce to Fig. 2. From observation of the top left parts of Figs. 17 to 19, a small $\beta$ function is found to be a preferred choice to generate small relative momentum compaction. Furthermore, the choice of $\psi_{21} \approx \pi$ (or its multiple integers) usually results in minimal $\left|R_{56}^{s_{1} \rightarrow s_{2}}\right|$, e.g. Figs. 17(a) and 17(b) except at $\alpha \approx 0$.

For a more general case of both dispersive and nonisochronous module, $R_{16}, R_{26}, R_{56} \neq 0$, the pattern of relative momentum compaction becomes more complicated, as shown in Figs. 20 and 21. The small $\beta$ function usually serves as a guideline to give small $\left|R_{56}^{s_{1} \rightarrow s_{2}}\right|$ [see Figs. 20(a) and 21(a)]. In addition, the choice of the small $\beta$ function provides more flexible parametric space of $\left|R_{56}^{s_{1} \rightarrow s_{2}}\right|$ than that of the larger $\beta$ function, although the larger $\beta$ function may still result in small $\left|R_{56}^{s_{1} \rightarrow s_{2}}\right|$ provided the betatron phase difference is properly chosen. As shown in Figs. 20(c) and 20(d), dependence of $\left|R_{56}^{s_{1} \rightarrow s_{2}}\right|$ on the $\alpha$ function can be weak. For larger dispersion, Figs. 21(c) and 21(d) indicate that moderate $\alpha$ functions can be preferred. 


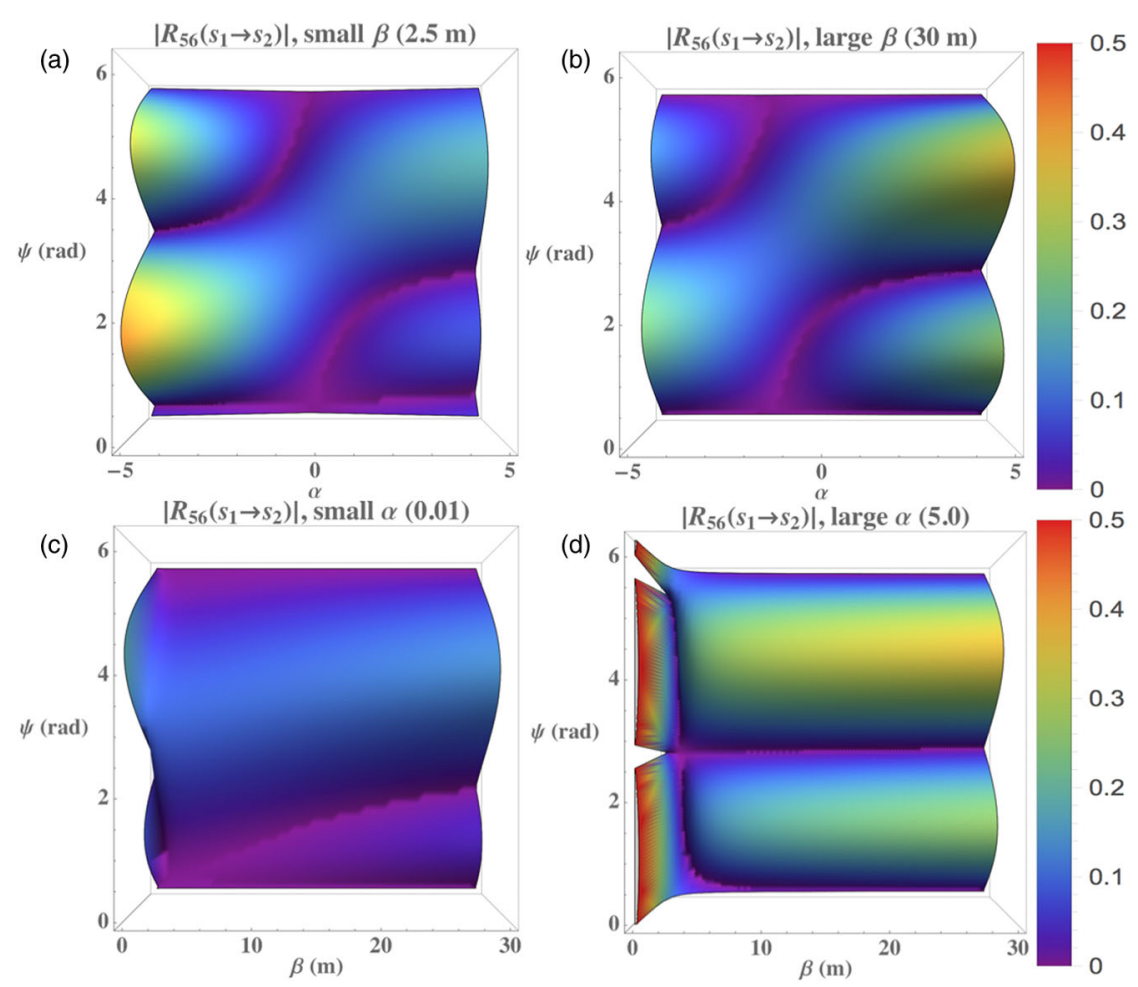

FIG. 17. $\left|R_{56}^{s_{1} \rightarrow s_{2}}\right|$ pattern for a dispersive but isochronous unit, $R_{16}=1 \mathrm{~m}, R_{26}=0, R_{56}=0$. (Top) $R_{56}^{s_{1} \rightarrow s_{2}}(\alpha, \psi)$ for (a) small and (b) large $\beta$ functions; (bottom) $R_{56}^{s_{1} \rightarrow s_{2}}(\beta, \psi)$ for (c) small and (d) large $\alpha$ functions.
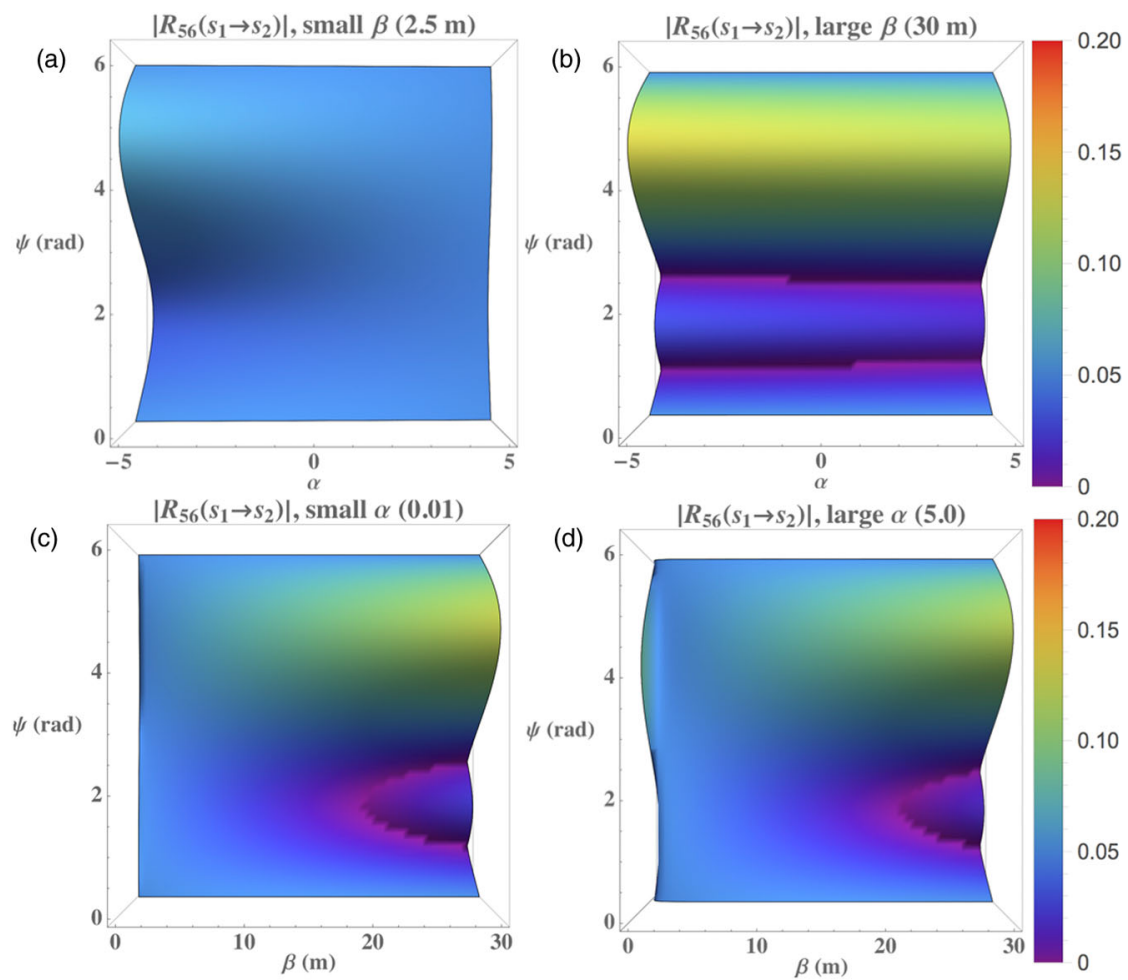

FIG. 18. $\left|R_{56}^{s_{1} \rightarrow s_{2}}\right|$ pattern for an achromatic but nonisochronous unit, $R_{16}=0, R_{26}=0, R_{56}=0.05 \mathrm{~m}$. (Top) $R_{56}^{s_{1} \rightarrow s_{2}}(\alpha, \psi)$ for (a) small and (b) large $\beta$ functions; (bottom) $R_{56}^{s_{1} \rightarrow s_{2}}(\beta, \psi)$ for (c) small and (d) large $\alpha$ functions. 

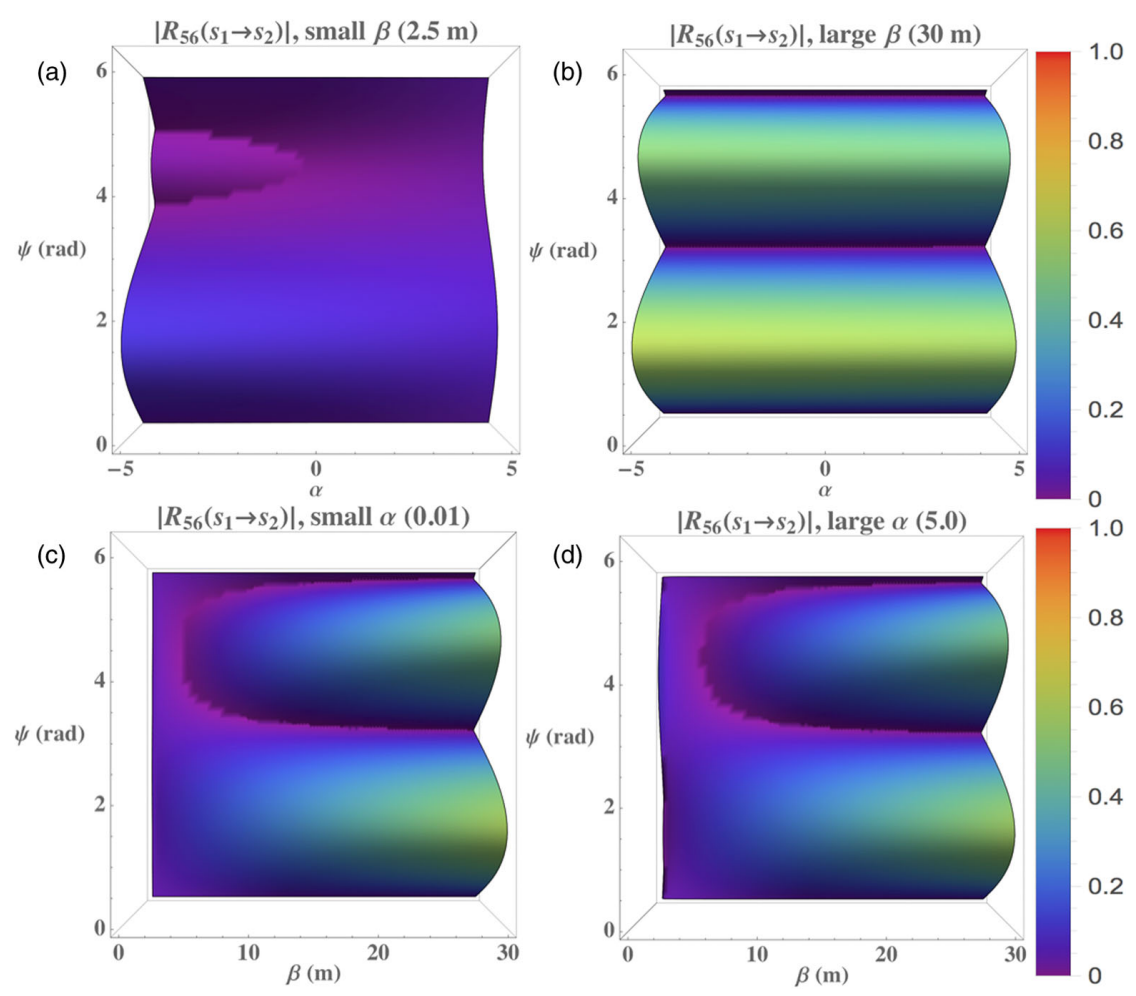

FIG. 19. $\left|R_{56}^{s_{1} \rightarrow s_{2}}\right|$ pattern for a dispersive and nonisochronous unit (small $\left.R_{26}\right), R_{16}=0, R_{26}=0.4, R_{56}=0.05 \mathrm{~m}$. (Top) $R_{56}^{s_{1} \rightarrow s_{2}}(\alpha, \psi)$ for (a) small and (b) large $\beta$ functions; (bottom) $R_{56}^{s_{1} \rightarrow s_{2}}(\beta, \psi)$ for (c) small and (d) large $\alpha$ functions.
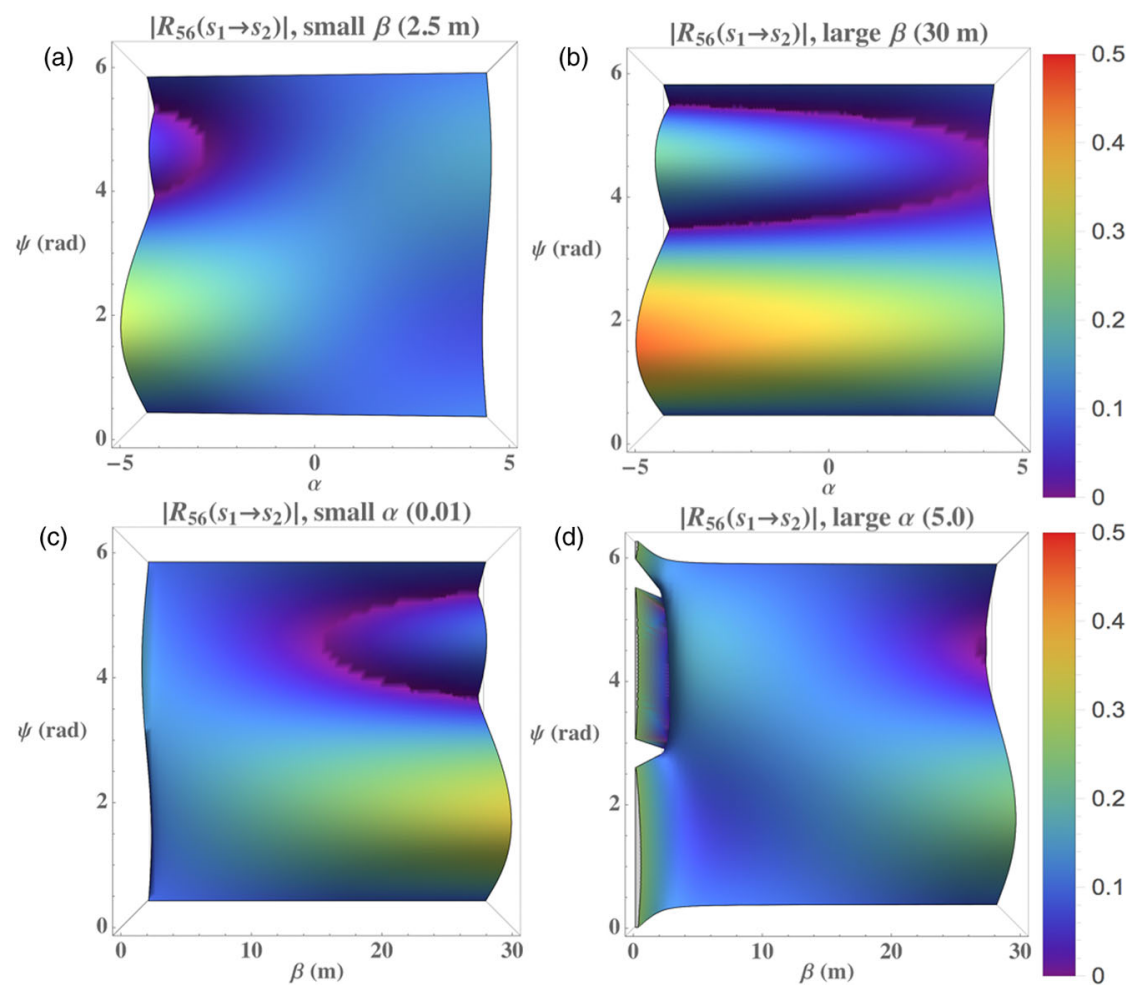

FIG. 20. $\left|R_{56}^{s_{1} \rightarrow s_{2}}\right|$ pattern for a dispersive and nonisochronous unit (small $R_{16}$ and $R_{26}$ ), $R_{16}=0.4 \mathrm{~m}, R_{26}=0.2, R_{56}=0.1 \mathrm{~m}$. (Top) $R_{56}^{s_{1} \rightarrow s_{2}}(\alpha, \psi)$ for (a) small and (b) large $\beta$ functions; (bottom) $R_{56}^{s_{1} \rightarrow s_{2}}(\beta, \psi)$ for (c) small and (d) large $\alpha$ functions. 

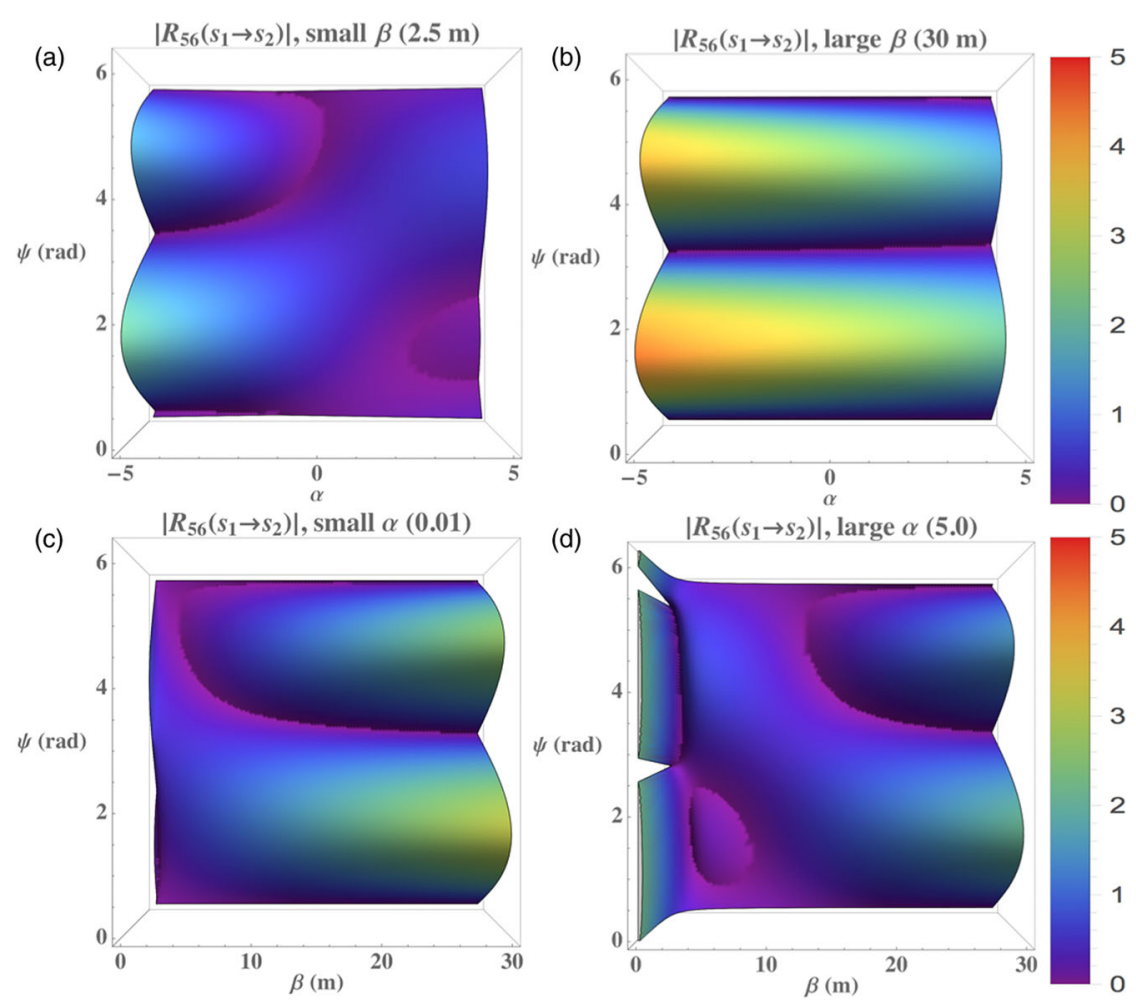

FIG. 21. $\left|R_{56}^{s_{1} \rightarrow s_{2}}\right|$ pattern for a dispersive and nonisochronous unit (large $R_{16}$ and $R_{26}$ ), $R_{16}=4 \mathrm{~m}, R_{26}=2, R_{56}=0.1 \mathrm{~m}$. (Top) $R_{56}^{s_{1} \rightarrow s_{2}}(\alpha, \psi)$ for (a) small and (b) large $\beta$ functions; (bottom) $R_{56}^{s_{1} \rightarrow s_{2}}(\beta, \psi)$ for (c) small and (d) large $\alpha$ functions.

Thus far we have already seen the parametric dependencies of $\left|R_{56}^{s_{1} \rightarrow s_{2}}\right|$ for a total of four typical cases: (i) achromatic and isochronous module $R_{16}=R_{26}=$ $R_{56}=0$ (Fig. 2); (ii) dispersive but isochronous module $R_{16} \neq 0, R_{26}=0, R_{56}=0$ (Fig. 17); (iii) achromatic but nonisochronous module (Fig. 18); and (iv) dispersive and nonisochronous module $R_{16}, R_{26}, R_{56} \neq 0$ of a beam line (Figs. 19 to 21). From the investigation of the $\left|R_{56}^{s_{1} \rightarrow s_{2}}\right|$ behavior, we conclude that small $\beta$ functions (at entrances and exits of dipoles), moderate $\alpha$ functions (at entrances and exits of dipoles), and $\psi_{21} \approx \pi$ (or its integer multiples) between two relevant dipoles can in general lead to a small relative momentum compaction function in a general transport line.

[1] M. Borland, Start-to-end jitter simulations of the Linac Coherent Light Source, in Proceedings of the 19th Particle Accelerator Conference, Chicago, IL, 2001 (IEEE, Piscataway, NJ, 2001), WPPH103.

[2] S. Heifets, G. Stupakov, and S. Krinsky, Coherent synchrotron radiation instability in a bunch compressor, Phys. Rev. ST Accel. Beams 5, 064401 (2002).

[3] Z. Huang and K.-J. Kim, Formulas for coherent synchrotron radiation microbunching in a bunch compressor chicane, Phys. Rev. ST Accel. Beams 5, 074401 (2002).
[4] D. Douglas, Suppression and enhancement of CSR-driven emittance degradation in the IR-FEL driver, Report No. JLAB-TN-98-012, 1998.

[5] R. Hajima, A first-order matrix approach to the analysis of electron beam emittance growth caused by coherent synchrotron radiation, Jpn. J. Appl. Phys. L974 (2003).

[6] S. Di Mitri, M. Cornacchia, and S. Spampinati, Cancellation of Coherent Synchrotron Radiation Kicks with Optics Balance, Phys. Rev. Lett. 110, 014801 (2013).

[7] Y. Jing, Y. Hao, and V.N. Litvinenko, Compensating effect of the coherent synchrotron radiation in bunch compressors, Phys. Rev. ST Accel. Beams 16, 060704 (2013).

[8] C. Mitchell, J. Qiang, and P. Emma, Longitudinal pulse shaping for the suppression of coherent synchrotron radiation-induced emittance growth, Phys. Rev. ST Accel. Beams 16, 060703 (2013).

[9] D. Douglas, S. V. Benson, A. Hutton, G. A. Krafft, R. Li, G. R. Neil, Y. Roblin, C. D. Tennant, and C.-Y. Tsai, Control of coherent synchrotron radiation, and microbunching effects during transport of high brightness electron beams, arXiv: 1403.2318v1; D. Douglas, S. V. Benson, A. Hofler, R. Kazimi, R. Li, Y. Roblin, C. D. Tennant, G. A. Krafft, B. Terzic, and C.-Y. Tsai, Control of synchrotron radiation effects during recirculation, in Proceedings of the 6th International Particle Accelerator Conference, Richmond, Virginia, TUPMA038 (2015), http://inspirehep.net/record/ 1395314.

[10] Y. Jiao, X. Cui, X. Huang, and G. Xu, Generic conditions for suppressing the coherent synchrotron radiation induced 
emittance growth in a two-dipole achromat, Phys. Rev. ST Accel. Beams 17, 060701 (2014).

[11] M. Venturini, CSR-induced emittance growth in achromats: Linear formalism revisited, Nucl. Instrum. Methods Phys. Res., Sect. A 794, 109 (2015).

[12] M. Venturini, Design of a triple-bend isochronous achromat with minimum coherent-synchrotron-radiationinduced emittance growth, Phys. Rev. Accel. Beams 19, 064401 (2016).

[13] E. L. Saldin, E. A. Schneidmiller, and M. V. Yurkov, Longitudinal space charge-driven microbunching instability in the TESLA Test Facility linac, Nucl. Instrum. Methods Phys. Res., Sect. A 528, 355 (2004).

[14] Z. Huang, M. Borland, P. Emma, J. Wu, C. Limborg, G. Stupakov, and J. Welch, Suppression of microbunching instability in the linac coherent light source, Phys. Rev. ST Accel. Beams 7, 074401 (2004).

[15] C. Behrens, Z. Huang, and D. Xiang, Reversible electron beam heating for suppression of microbunching instabilities at free-electron lasers, Phys. Rev. ST Accel. Beams 15, 022802 (2012).

[16] J. Qiang, C. Mitchell, and M. Venturini, Suppression of Microbunching Instability Using Bending Magnets in Free-Electron-Laser Linacs, Phys. Rev. Lett. 111, 054801 (2013).

[17] S. Di Mitri and S. Spampinati, Microbunching Instability Suppression via Electron-Magnetic-Phase Mixing, Phys. Rev. Lett. 112, 134802 (2014).

[18] C.-Y. Tsai, D. Douglas, R. Li, and C. Tennant, Linear microbunching analysis for recirculation machines, Phys. Rev. ST Accel. Beams 19, 114401 (2016).

[19] Y.S. Derbenev, J. Rossbach, E. L. Saldin, and V. D. Shiltsev, Microbunch radiative tailhead interaction, TESLA-FEL Report No. 1995-05.

[20] J. B. Murphy, S. Krinsky, and R. L. Gluckstern, Longitudinal wakefield for an electron moving on a circular orbit, Part. Accel. 57, 9 (1997).

[21] C.-Y. Tsai, D. Douglas, R. Li, and C. Tennant, Multistage CSR microbunching gain development in transport or recirculation arcs, in Proceedings of 37th International Free Electron Laser Conference, Daejeon, Korea, MOP087.

[22] K. L. Brown, A first and second order matrix theory for the design of beam transport systems and charged particle spectrometers, Adv. Part. Phys. 1, 71 (1968); Report No. SLAC-75 Rev.4, 1982.

[23] S. Y. Lee, Accelerator Physics, 2nd ed. (World Scientific, Singapore, 2004).

[24] M. Borland, Modeling of microbunching instability, Phys. Rev. ST Accel. Beams 11, 030701 (2008).

[25] M. Borland, Controlling noise and choosing binning parameters for reliable CSR and LSC simulation in elegant, Report No. OAG-TN-2005-027.
[26] C.-Y. Tsai and R. Li, Simulation of coherent synchrotron radiation induced microbunching gain using ELEGANT, Report No. JLAB-TN-14-016.

[27] E. L. Saldin, E. A. Schneidmiller, and M. V. Yurkov, On the coherent radiation of an electron bunch moving in an arc of a circle, Nucl. Instrum. Methods Phys. Res., Sect. A 398, 373 (1997).

[28] R. A. Bosch, Longitudinal wake of a bunch of suddenly accelerated electrons within the radiation formation zone, Phys. Rev. ST Accel. Beams 10, 050701 (2007).

[29] P. Emma and G. Stupakov, CSR wake for a short magnet in ultrarelativistic limit, in Proceedings of the 8th European Particle Accelerator Conference, Paris, 2002 (EPS-IGA and CERN, Geneva, 2002), pp. 1479.

[30] C.-Y. Tsai and R. Li, Combination of density and energy modulation in microbunching analysis, in Proceedings of the 7th International Particle Accelerator Conference, TUPOR020.

[31] C.-Y. Tsai, Y. Derbenev, D. Douglas, R. Li, and C. Tennant, Vlasov analysis of microbunching instability for magnetized beams (to be published).

[32] S. Di Mitri, Intrabeam scattering in high brightness electron linacs, Phys. Rev. ST Accel. Beams 17, 074401 (2014).

[33] D. R. Douglas, S. V. Benson, R. Li, Y. Roblin, C. D. Tennant, G. A. Krafft, B. Terzic, and C.-Y. Tsai, Control of synchrotron radiation effects during recirculation with bunch compression, in Proceedings of the 6th International Particle Accelerator Conference, TUPMA037.

[34] When the modulation wavelength becomes longer, we notice that the free-space CSR impedance models may be invalid when the wall shielding effect becomes important. In that case the radiation shielding should be taken into account when the distance from the beam orbit to the walls $h / 2$ satisfies $h \leq\left(\rho \lambda^{2}\right)^{1 / 3}$. In addition, when the modulation wavelength is comparable to the bunch length, the coasting beam model is no longer valid and the finite bunched beam model shall be employed in the microbunching analysis of the long wavelength regime.

[35] E. L. Saldin, E. A. Schneidmiller, and M. V. Yurkov, Klystron instability of a relativistic electron beam in a bunch compressor, Nucl. Instrum. Methods Phys. Res., Sect. A 490, 1 (2002).

[36] C.-Y. Tsai, D. Douglas, R. Li, and C. Tennant, Linear microbunching gain estimation including CSR and LSC impedances in recirculation machines, in Proceedings of the 56th ICFA Advanced Beam Dynamics Workshop on Energy Recovery Linacs, Stony Brook, New York, USA, TUICLH2034.

[37] M. Venturini and J. Qiang, Transverse space-charge induced microbunching instability in high-brightness electron bunches, Phys. Rev. ST Accel. Beams 18, 054401 (2015). 